\title{
SOME REGULARITY RESULTS FOR A CLASS OF UPPER SEMICONTINUOUS FUNCTIONS
}

\author{
ANTONIO MARIGONDA, KHAI T. NGUYEN, AND DAVIDE VITTONE
}

\begin{abstract}
We study regularity properties enjoyed by a class of real-valued upper semicontinuous functions $f: \mathbb{R}^{d} \rightarrow \mathbb{R}$ whose hypograph satisfies a geometric property implying, for each point $P$ on the boundary of hypo $f$, the existence of a sort of (uniform) subquadratic tangent hypersurface whose intersection with hypo $f$ in a neighbourhood of $P$ reduces to $P$. This geometric property generalizes both the concepts of semiconcave functions and functions whose hypograph has positive reach in the sense of Federer; the associated class of functions arises in the study of regularity properties for the minimum time function of certain classes of nonlinear control systems and differential inclusions.

We will prove that these functions share several regularity properties with semiconcave functions. In particular, they are locally $B V$ and differentiable a.e. Our approach consists in providing upper bounds for the dimension of the set of nondifferentiability points. Moreover, a finer classification of the singularities can be performed according to the dimension of the normal cone to the hypograph, thus generalizing a similar result proved by Federer for sets with positive reach. Techniques of nonsmooth analysis and geometric measure theory are used.
\end{abstract}

\section{INTRODUCTION}

We study a class of upper semicontinuous functions $f: \mathbb{R}^{d} \rightarrow \mathbb{R}$ whose hypograph hypo $f$ (see Definition 2.3) satisfies a geometric regularity property, namely: there exist $c>0, \theta \in] 0,1]$ such that for each $P$ on the boundary of hypo $f$ there exists a unitary Fréchet (outer) normal $v \in N_{\text {hypo } f}^{F}(P) \cap \mathbb{S}^{d}$ to hypo $f$ with

$$
\langle v, P-Q\rangle \leq c\|P-Q\|^{1+\theta} \quad \text { for every } Q \in \operatorname{hypo} f .
$$

Geometrically speaking, this inequality expresses the fact that, in a neighborhood of each point $P$ on the boundary of hypo $f$, there exists a "subquadratic" smooth hypersurface $\Gamma(P)$ whose intersection with hypo $f$ reduces to $P$. One could also say that $\Gamma(P)$ is supertangent to hypo $f$ in a generalized sense.

Date: November 29, 2011.

1991 Mathematics Subject Classification. 49J52; 26B30.

Key words and phrases. exterior sphere condition, sets with positive reach, reduced boundary.

The authors are supported by MIUR, GNAMPA of INDAM, Fondazione CaRiPaRo Project "Nonlinear Partial Differential Equations: models, analysis, and control-theoretic problems" and University of Padova research project "Some analytic and differential geometric aspects in Nonlinear Control Theory, with applications to Mechanics". A.M. is also supported by University of Verona. K.T.N is also supported by the ERC Starting Grant 2009 n.240385 ConLaws. K.T.N and D.V. are also supported by University of Padova. 
When $\theta=1$ condition (1) reads as

$$
\left\|\left(P-\frac{v}{2 c}\right)-Q\right\| \geq \frac{1}{2 c} \quad \text { for every } Q \in \operatorname{hypo} f,
$$

which means that the open sphere of center $P-\frac{v}{2 c}$ and radius $\frac{1}{2 c}$ lies outside hypo $f$ and touches the boundary of hypof at $P$. This property is also called exterior sphere condition and was studied by several authors, mainly in connection with regularity problems arising in the control theory. In particular, in Proposition 3.2 of [9] it is proved that if a closed set $K \subseteq \mathbb{R}^{d+1}$ satisfies an interior sphere condition (i.e., the closure of its complement satisfies an exterior sphere condition), then the distance function dist $(\cdot, K)$ satisfies in $\overline{\mathbb{R}^{d+1} \backslash K}$ a regularity property called semiconcavity with a linear modulus, which can be regarded as a smooth $C^{2}$ perturbation of concavity. We refer the reader to the monograph [10] for a detailed description of the properties of semiconcave functions and their applications to the regularity theory for the value function of optimal control problems.

If we strenghten the exterior sphere condition by requiring (2) to hold for every $v \in N_{\text {hypo } f}^{F}(P) \cap$ $\mathbb{S}^{d}$ (while in its formulation this is required just for at least one normal) we are in the class of functions whose hypograph has positive reach in the sense of Federer. In finite dimension, sets of positive reach were introduced by Federer in [24] as a generalization of convex sets and sets with $C^{2}$-boundary. They enjoy several strong geometrical characterization, the following statements being indeed equivalent:

i. $K \subseteq \mathbb{R}^{d+1}$ is a closed set with positive reach;

ii. property (2) holds for every $v \in N_{\text {hypo } f}^{F}(P) \cap \mathbb{S}^{d}$;

iii. there exists a neighborhood $U$ of $K$ such that $\operatorname{dist}(\cdot, K)$ is of class $C^{1,1}(U)$;

iv. there exists a neighborhood $U$ of $K$ such that the metric projection onto $K$ is singlevalued.

If we are also allowed to take $C=0$ in condition (1), then the set is convex and $U=\mathbb{R}^{d+1}$. Several authors studied sets with positive reach in both finite and infinite dimension; we refer to [21] for a comprehensive summary of the results on this topic.

Upper semicontinuous functions whose hypograph has positive reach share several regularity properties with concave functions: it was proved in [15] that around a.e. points of their domain they are actually Lipschitz continuous, semiconcave with linear modulus and twice differentiable a.e. In [17], [19] and [20] some regularity results were proved for the minimum time function of control problems; under suitable weak controllability assumptions, the latter is proved to have epigraph or hypograph with locally positive reach, thus generalizing the results of [9] and [10].

The link between the exterior sphere condition and the positive reach property was recently investigated in a series of paper [32-36] where several properties and sufficient conditions granting positive reach properties are proven starting from the weaker exterior sphere condition. One of the main results in this sense is that if a set satisfies an exterior sphere condition and it is wedged (i.e., the normal cone does not contain lines) then it has positive reach. From a different viewpoint, it was shown in $[30,31]$ that the notions of exterior sphere and positive reach 
are almost equivalent in the sense of measure: namely, up to a closed exceptional set of zero measure, every set satisfying a uniform exterior sphere condition has positive reach.

However, it is easy to give examples where the hypograph of the minimum time function does not satisfy an exterior sphere property, so that the results of $[19,30]$ can not be applied. Let us consider the constant control system

$$
\left\{\begin{array}{l}
\dot{x}(t)=0, \\
\dot{y}(t)=u(t) \in[0,1], \\
(x(0), y(0))=\left(x_{0}, y_{0}\right) \in \mathbb{R}^{2},
\end{array}\right.
$$

together with the target $\mathcal{T}=\{(x, \beta) \mid \beta \geq f(x)\}$, where

$$
f(x):= \begin{cases}1 & \text { if } x \leq 0 \\ -x^{\frac{2}{3}} & \text { if } x>0 .\end{cases}
$$

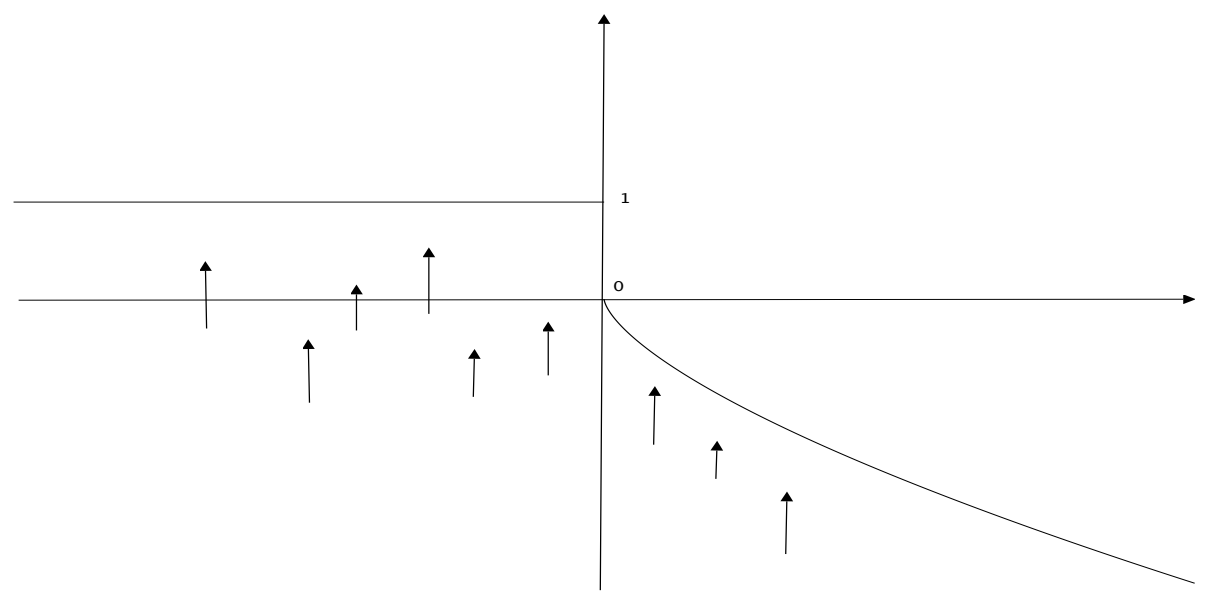

FiguRE 1 . The system $\dot{x}=0, \dot{y} \in[0,1]$ and the target $\mathcal{T}$.

The minimum time to reach the target $\mathcal{T}$ subject to the above control system is denoted by $T$. It can be proved (see the Appendix) that hypo $T$ does not satisfy an exterior sphere condition, but still enjoys the weaker uniformity regularity property (1) with $\theta=1 / 2$.

The previous considerations motivate us to study the class $\mathscr{F}(\Omega)$ of real functions defined on $\Omega \subset \mathbb{R}^{d}$ satisfying condition (1) in order to provide a new regularity class which, hopefully, will cover the regularity properties for the minimum time function of certain classes of nonlinear control systems and differential inclusions [11] that does not satisfy an exterior sphere condition.

We state our first general result for closed set $K \subset \mathbb{R}^{d+1}$ concerning the structure and dimension of the set $K^{(j)}$ of points on $\partial K$ where the Fréchet normal cone to $\partial K$ has dimension larger than or equal to $j$. This result generalizes a similar result proved by Federer for sets with positive reach. Indeed, it shows that $K^{(j)}$ can be covered by countably many Lipschitz graphs of $d-j+1$ variables. 
Theorem 1.1. Let $K \subseteq \mathbb{R}^{d+1}$ be closed; then $K^{(j)}$ is countably $\mathscr{H}^{d-j+1}$-rectifiable. In particular, also $K_{ \pm}^{(j)}$ are countably $\mathscr{H}^{d-j+1}$-rectifiable.

The sets $K_{ \pm}^{(j)}$ are here defined in the same way of $K^{(j)}$ by taking the normal cone to, respectively, $K$ and $\overline{\mathbb{R}^{d+1} \backslash K}$; see Definition 4.1. Concerning the differentiability properties of functions, we denote by $\mathscr{S}_{f}$ the set of non-differentiability points of $f$ and prove the following result:

Theorem 1.2. Let $\Omega$ be a nonempty open subset of $\mathbb{R}^{d}$ and let $f \in B V_{\text {loc }}(\Omega)$ be an upper semicontinuous function; set $K:=$ hypo $f$. Assume that for $\mathscr{H}^{d}$-a.e. $\left(x, \beta_{x}\right) \in \partial K \cap(\Omega \times \mathbb{R})$ it holds $N_{K}^{F}\left(x, \beta_{x}\right) \neq\{0\}$. Then $\mathscr{L}^{d}\left(\mathscr{S}_{f}\right)=0$.

The previous result is applied to show that functions in the class $\mathscr{F}(\Omega)$ will share several properties with semiconcave functions with a nonlinear modulus like having (locally) bounded variation and being differentiable a.e. Moreover, for function in $\mathscr{F}(\Omega)$ finer $B V$ estimates can be performed around singular points; such estimates give sharp upper bounds, related to the exponent $\theta$ appearing in (1), on the dimension of $\mathscr{S}_{f}$.

Theorem 1.3. Let $\Omega \subseteq \mathbb{R}^{d}$ be nonempty and open and let $f \in \mathscr{F}(\Omega)$. Then for any open set $U \subset \subset \Omega$ we have

In particular, $\operatorname{dim}_{\mathscr{H}} \mathscr{S}_{f} \leq d-\frac{\theta}{1+\theta}$.

$$
\mathscr{H}^{d-\frac{\theta}{1+\theta}}\left(\mathscr{S}_{f} \cap U\right)<+\infty .
$$

There is still an open problem, arising in the study of the minimum time function, to provide suitable sufficient conditions yielding $S B V$ regularity; indeed, as showed in [18], in general this property does not hold even in the positive reach case.

The paper is organized as follows. In Section 2 we fix the notation and state definitions and preliminary known results of nonsmooth analysis and geometric measure theory that will be used later. In Section 3 we introduce the main objects of our investigations and discuss their simplest properties. Section 4 and 5 are devoted to the proofs and consequences of, respectively, Theorems 1.1 and 1.2. In Section 6 we give sufficient conditions on functions ensuring the local semiconcavity property out of the singular set and perform a comparison between the Frechét and measure theoretic normals to sets. In Section 7 we prove Theorem 1.3. Finally, in the Appendix we discuss an example arising in the minimum time problem.

\section{Preliminaries AND NOtation}

We begin by recalling some basic notation.

Definition 2.1. Let $K$ be a closed subset of $\mathbb{R}^{d}, S \subseteq \mathbb{R}^{d}, x=\left(x_{1}, \ldots, x_{d}\right) \in K, y=$ $\left(y_{1}, \ldots, y_{d}\right) \in \mathbb{R}^{d}, r>0$. We denote by

- $\langle\cdot, \cdot\rangle$, the usual scalar product in $\mathbb{R}^{d}$;

- $\partial S, \operatorname{int}(S), \bar{S}$, the topological boundary, interior and closure of $S$, respectively;

- $\operatorname{diam}(S):=\sup \left\{\left\|z_{1}-z_{2}\right\|: z_{1}, z_{2} \in S\right\}$, the diameter of $S$;

- $\mathcal{P}(S):=\left\{B \subseteq \mathbb{R}^{d}: B \subseteq S\right\}$, the power set of $S$; 
- $\mathbb{B}^{d}:=\left\{w \in \mathbb{R}^{d}:\|w\|<1\right\}$, the unit open ball (centered at the origin);

. $\mathbb{S}^{d-1}:=\left\{w \in \mathbb{R}^{d}:\|w\|=1\right\}=\partial \mathbb{B}^{d}$, the unit sphere (centered at the origin);

- $B(y, r):=\left\{z \in \mathbb{R}^{d}:\|z-y\|<r\right\}=y+r \mathbb{B}^{d}$, the open ball of center $y$ and radius $r$;

- $\operatorname{Sq}(y, r):=\left\{\left(z_{1}, \ldots, z_{d}\right) \in \mathbb{R}^{d}: \max _{i=1, \ldots, d}\left|y_{i}-z_{i}\right|<r\right\}$, the open square of center $y$ and side $2 r$;

- $d_{K}(y):=\operatorname{dist}(y, K)=\min \{\|z-y\|: z \in K\}$, the distance of $y$ from $K$;

- $\pi_{K}(y):=\left\{z \in K:\|z-y\|=d_{K}(y)\right\}$, the set of projections of $y$ onto $K$.

If $\pi_{K}(y)=\{\xi\}$, i.e. it is a singleton, we will identify the set $\pi_{K}(y)$ with its unique element and write $\pi_{K}(y)=\xi$.

The characteristic function $\chi_{S}: \mathbb{R}^{d} \rightarrow\{0,1\}$ of $S$ is defined as $\chi_{S}(x)=1$ if $x \in S$ and $\chi_{S}(x)=0$ if $x \notin S$.

If $S_{1}, S_{2} \subseteq \mathbb{R}^{d}$, their symmetric difference is defined as $S_{1} \triangle S_{2}=\left(S_{1} \cup S_{2}\right) \backslash\left(S_{1} \cap S_{2}\right)$.

If $V, W \subseteq \mathbb{R}^{d}$ are two subset of $\mathbb{R}^{d}$, we will write $V \subset \subset W$ if $V$ is bounded and $\bar{V} \subseteq W$.

The Fréchet normal cone and the Bouligand tangent cone to $K$ at $x$ are defined respectively by

$$
\begin{aligned}
& N_{K}^{F}(x):=\left\{v \in \mathbb{R}^{d}: \limsup _{\substack{y \rightarrow x \\
y \in K \backslash\{x\}}}\left\langle v, \frac{y-x}{\|y-x\|}\right\rangle \leq 0\right\} ; \\
& T_{K}^{F}(x):=\left\{\lambda \xi \in \mathbb{R}^{d}: \lambda \geq 0, \exists\left\{y_{n}\right\}_{n} \subseteq K \backslash\{x\}, y_{n} \rightarrow x \text { s.t. } \xi=\lim _{n \rightarrow \infty} \frac{y_{n}-x}{\left\|y_{n}-x\right\|}\right\} .
\end{aligned}
$$

Notice that $N_{K}^{F}(x)$ is always closed and convex. We have

$$
N_{K}^{F}(x)=\left(T_{K}^{F}(x)\right)^{*}:=\left\{v \in \mathbb{R}^{d}:\langle v, w\rangle \leq 0 \text { for all } w \in T_{K}^{F}(x)\right\} .
$$

Definition 2.2. Let $V, W \subseteq \mathbb{R}^{d+1}$ be nonempty. The vector space generated by $V$ is

$$
\operatorname{Span}(V):=\left\{\sum_{j=1}^{n} a_{j} v_{j}: n \in \mathbb{N}, a_{j} \in \mathbb{R}, v_{j} \in V, j=1, \ldots, n\right\} .
$$

The set $W$ is called convex if for every $w_{1}, w_{2} \in W, \lambda \in[0,1]$, we have $\lambda w_{1}+(1-\lambda) w_{2} \in W$. We denote by $\operatorname{dim} W$ the dimension of the linear space $\operatorname{Span}(W-W)$ spanned by the elements of $W-W:=\left\{w_{1}-w_{2}: w_{1}, w_{2} \in W\right\}$, and notice that $\operatorname{Span}(W-W)=\operatorname{Span}(W)$ if $0 \in W$. 
Definition 2.3. Let $\Omega \subseteq \mathbb{R}^{d}$ and $f: \Omega \rightarrow \mathbb{R} \cup\{ \pm \infty\}$ be a function. For $x \in \Omega$ fixed we denote by

$$
\begin{aligned}
\bar{f}(x) & :=\limsup _{\substack{y \rightarrow x \\
y \neq x}} f(y), \quad \widetilde{f}(x):=\limsup _{y \rightarrow x} f(y)=\max \{f(x), \bar{f}(x)\} \\
\underline{f}(x) & :=\liminf _{\substack{y \rightarrow x \\
y \neq x}} f(y), \quad \mathcal{\sim}_{\sim}(x):=\liminf _{y \rightarrow x} f(y)=\min \{f(x), \underline{f}(x)\} \\
\operatorname{dom}(f) & :=\{z \in \Omega: f(z) \in \mathbb{R}\}, \text { the domain of } f ; \\
\text { hypo } f & :=\{(z, \beta) \in \Omega \times \mathbb{R}: \beta \leq f(z)\}, \text { the hypograph of } f ; \\
\text { epi } f & :=\{(z, \alpha) \in \Omega \times \mathbb{R}: \alpha \geq f(z)\}, \text { the epigraph of } f ; \\
\partial^{F} f(x) & :=\left\{v \in \mathbb{R}^{d}:(-v, 1) \in N_{\text {hypo } f}^{F}(x, f(x))\right\} ; \\
\partial_{F} f(x) & :=\left\{v \in \mathbb{R}^{d}:(v,-1) \in N_{\text {epi } f}^{F}(x, f(x))\right\} .
\end{aligned}
$$

We say that $f$ is upper (respectively, lower) semicontinuous if $f(x) \geq \bar{f}(x)$ (resp., if $f(x) \leq \underline{f}(x)$ ) for any $x \in \Omega$. Equivalently, if hypo $f$ (resp., epi $f$ ) is relatively closed in $\Omega \times \mathbb{R}$. For u.s.c. functions it holds

$$
\underset{\sim}{f}(x)=\underline{f}(x) \leq \bar{f}(x) \leq f(x)=\widetilde{f}(x) \quad \forall x \in \Omega
$$

while for l.s.c. functions one has

$$
\underset{\sim}{f}(x)=f(x) \leq \underline{f}(x) \leq \bar{f}(x)=\widetilde{f}(x) \quad \forall x \in \Omega .
$$

The sets $\partial^{F} f(x)$ and $\partial_{F} f(x)$ are called respectively the Fréchet superdifferential and the Fréchet subdifferential of $f$ at $x$. We recall that

$$
\begin{aligned}
& \partial_{F} f(x)=\left\{v \in \mathbb{R}^{d}: \liminf _{y \rightarrow x} \frac{f(y)-f(x)-\langle v, y-x\rangle}{\|y-x\|} \geq 0\right\} ; \\
& \partial^{F} f(x)=\left\{v \in \mathbb{R}^{d}: \limsup _{y \rightarrow x} \frac{f(y)-f(x)-\langle v, y-x\rangle}{\|y-x\|} \leq 0\right\}
\end{aligned}
$$

are respectively the set of Fréchet subgradients and supergradients of $f$ at $x$. If $\partial_{F} f(x)$ contains more than one element, we have that $\partial^{F} f(x)=\emptyset$ and conversely if $\partial^{F} f(x)$ contains more than one element, we have that $\partial_{F} f(x)=\emptyset$. It may happen that $\partial_{F} f(x)=\partial^{F} f(x)=\emptyset$. We have that $f$ is differentiable at $x$, with differential denoted by $\nabla f(x)$, if and only if $\partial_{F} f(x)$ and $\partial^{F} f(x)$ are both nonempty; in this case $\partial_{F} f(x)=\partial^{F} f(x)=\{\nabla f(x)\}$.

For the sake of completeness we state and prove the following simple results, which we will use several times throughout the paper.

Lemma 2.4. Let $\Omega$ be a nonempty open subset of $\mathbb{R}^{d}$ and $f: \Omega \rightarrow \mathbb{R}$ be a function. Set $K:=\operatorname{hypo} f$. Then

(i) if $(x, \beta) \in \partial K$ and $v \in N_{K}^{F}(x, \beta)$, then $v_{d+1} \geq 0$;

(ii) for all $x \in \Omega$ we have $(x, \beta) \in \partial K \Leftrightarrow \underset{\sim}{f}(x) \leq \beta \leq \widetilde{f}(x)$;

(iii) if $\underset{\sim}{f}(x)<\beta<\widetilde{f}(x)$ we have $N_{\partial K}^{F}(x, \beta) \subseteq \mathbb{R}^{d} \times\{0\}$; 
(iv) if $\underset{\sim}{f}(x) \leq \beta<\widetilde{f}(x)$ we have $N_{K}^{F}(x, \beta) \subseteq \mathbb{R}^{d} \times\{0\}$;

(v) if $\underset{\sim}{f}(x) \leq \beta_{1}<\beta_{2} \leq \widetilde{f}(x)$, then $N_{K}^{F}\left(x, \beta_{1}\right) \subseteq N_{K}^{F}\left(x, \beta_{2}\right)$.

Proof. Let us begin with statement (i). Let $\left\{\left(x_{k}, \beta_{k}\right)\right\}_{k \in \mathbb{N}} \subseteq K$ be a sequence converging to $(x, \beta) \in \partial K$. Since

$$
\gamma_{k}:=\min \left\{\beta, \beta_{k}\right\}-\left\|x_{k}-x\right\|^{1 / 2}<\beta_{k} \leq f\left(x_{k}\right)
$$

we have also $\left(x_{k}, \gamma_{x}\right) \in K$ for any $k$. Moreover, one has $\left(x_{k}, \gamma_{k}\right) \rightarrow(x, \beta)$ and

$$
\left\|x_{k}-x\right\|=o\left(\left\|x_{k}-x\right\|^{1 / 2}\right) \leq o\left(\left|\gamma_{k}-\beta\right|\right)
$$

(we have used $\left|\gamma_{k}-\beta\right|=\beta-\gamma_{k} \geq\left\|x_{k}-x\right\|^{1 / 2}$ ) and this gives

$$
-v_{d+1}=\lim _{k \rightarrow \infty}\left\langle v, \frac{\left(x_{k}, \gamma_{k}\right)-(x, \beta)}{\left\|\left(x_{k}, \gamma_{k}\right)-(x, \beta)\right\|}\right\rangle \leq 0
$$

because $v \in N_{K}^{F}(x, \beta)$.

We now examine statement (ii). If $\beta<\underset{\sim}{f}(x)$ (respectively, $\beta>\widetilde{f}(x)$ ) it is easy to show that $(x, \beta) \in \operatorname{int} K$ (resp., $\left.(x, \beta) \in \operatorname{int}\left(\mathbb{R}^{d+1} \backslash K\right)\right)$. This proves one implication. For the reverse one, fix $x \in \Omega$ and let $\left\{\underline{x}_{k}\right\}_{k \in \mathbb{N}},\left\{\bar{x}_{k}\right\}_{k \in \mathbb{N}}$ be two sequences in $\Omega$ converging to $x$ and such that

$$
f\left(\bar{x}_{k}\right) \rightarrow \widetilde{f}(x), \quad f\left(\underline{x}_{k}\right) \rightarrow \underset{\sim}{f}(x) \quad \text { as } k \rightarrow+\infty .
$$

Take $\underset{\sim}{f}(x) \leq \beta \leq \widetilde{f}(x), \beta \in \mathbb{R}$; possibly passing to a (not relabeled) subsequence we have for large enough $k$

$$
f\left(\underline{x}_{k}\right)<\beta+\frac{1}{k} \quad \text { and } \quad \beta-\frac{1}{k}<f\left(\bar{x}_{k}\right),
$$

i.e.,

$$
\left(\underline{x}_{k}, \beta+\frac{1}{k}\right) \notin K, \quad\left(\bar{x}_{k}, \beta-\frac{1}{k}\right) \in K .
$$

This gives $(x, \beta) \in \partial K$ because both $\left(\underline{x}_{k}, \beta+\frac{1}{k}\right)$ and $\left(\bar{x}_{k}, \beta-\frac{1}{k}\right)$ converge to $(x, \beta)$.

Concerning statement (iii), we want to prove that, if $v=\left(v^{\prime}, v_{d+1}\right) \in \mathbb{R}^{d} \times \mathbb{R}$ is such that $v_{d+1} \neq 0$, then $v \notin N_{\partial K}^{F}(x, \beta)$. If $\varepsilon>0$ is small enough we have $\left.\beta+\varepsilon \operatorname{sgn}\left(v_{d+1}\right) \in\right] f(x), \widetilde{f}(x)[$ and, by statement (ii),

$$
\left(x, \beta+\varepsilon \operatorname{sgn}\left(v_{d+1}\right)\right) \in \partial K .
$$

Thus

$$
\lim _{\varepsilon \rightarrow 0^{+}}\left\langle v, \frac{\left(x, \beta+\varepsilon \operatorname{sgn}\left(v_{d+1}\right)\right)-(x, \beta)}{\left\|\left(x, \beta+\varepsilon \operatorname{sgn}\left(v_{d+1}\right)\right)-(x, \beta)\right\|}\right\rangle=\left|v_{d+1}\right|>0,
$$

i.e., $v \notin N_{\partial K}^{F}(x, \beta)$.

As for statement (iv), we have by (i) that $v_{d+1} \geq 0$ for any $v \in N_{K}^{F}(x, \beta), \beta \in[\underset{\sim}{f}(x), \widetilde{f}(x)[$. Since $(x, \beta+\varepsilon) \in K$ for $\varepsilon>0$ small enough one has

$$
v_{d+1}=\lim _{\varepsilon \rightarrow 0^{+}}\left\langle v, \frac{(x, \beta+\varepsilon)-(x, \beta)}{\|(x, \beta+\varepsilon)-(x, \beta)\|}\right\rangle \leq 0,
$$

whence $v_{d+1}=0$, as desired. 
Finally, statement (iv) ensures that, if $v \in N_{K}^{F}\left(x, \beta_{1}\right)$ and $\beta_{1}<\beta_{2}$, then $v_{d+1}=0$. Therefore

$$
\begin{aligned}
\limsup _{K \ni(y, \gamma) \rightarrow\left(x, \beta_{2}\right)}\left\langle v, \frac{(y, \gamma)-\left(x, \beta_{2}\right)}{\left\|(y, \gamma)-\left(x, \beta_{2}\right)\right\|}\right\rangle & =\limsup _{K \ni(y, \gamma) \rightarrow\left(x, \beta_{2}\right)}\left\langle v, \frac{\left(y, \gamma-\left(\beta_{2}-\beta_{1}\right)\right)-\left(x, \beta_{1}\right)}{\left\|\left(y, \gamma-\left(\beta_{2}-\beta_{1}\right)\right)-\left(x, \beta_{1}\right)\right\|}\right\rangle \\
& \leq \limsup _{K \ni(y, \tilde{\gamma}) \rightarrow\left(x, \beta_{1}\right)}\left\langle v, \frac{(y, \tilde{\gamma})-\left(x, \beta_{1}\right)}{\left\|(y, \tilde{\gamma})-\left(x, \beta_{1}\right)\right\|}\right\rangle \leq 0,
\end{aligned}
$$

i.e., $v \in N_{K}^{F}\left(x, \beta_{2}\right)$. We have used the fact that

$$
\begin{aligned}
& (y, \gamma) \rightarrow\left(x, \beta_{2}\right) \Longleftrightarrow(y, \tilde{\gamma}):=\left(y, \gamma-\left(\beta_{2}-\beta_{1}\right)\right) \rightarrow\left(x, \beta_{1}\right) \\
& (y, \gamma) \in K \Longrightarrow(y, \tilde{\gamma}):=\left(y, \gamma-\left(\beta_{2}-\beta_{1}\right)\right) \in K,
\end{aligned}
$$

This concludes the proof of statement $(\mathrm{v})$ and of the Lemma.

Definition 2.5. Let $C \subseteq \mathbb{R}^{d+1}$ and $N: C \rightarrow \mathcal{P}\left(\mathbb{R}^{d+1}\right)$ be a set valued map, which will be also called a multifunction and denoted by $N: C \rightrightarrows \mathbb{R}^{d+1}$. We say that $N$ has closed graph if, for every sequence $\left\{\left(x_{n}, v_{n}\right)\right\}_{n \in \mathbb{N}} \subseteq C \times \mathbb{R}^{d+1}$ converging to $(x, v) \in C \times \mathbb{R}^{d+1}$ and such that $v_{n} \in N\left(x_{n}\right)$ for every $n \in \mathbb{N}$, we have $v \in N(x)$.

A multifunction $N: C \rightrightarrows \mathbb{R}^{d+1}$ is upper semicontinuous if for every $x \in C$ and $c=c_{x}>0$ there exists $\delta=\delta\left(c_{x}, x\right)>0$ such that $N(y) \subseteq N(x)+c_{x} \mathbb{B}^{d+1}$ for every $y \in C \cap\left(x+\delta\left(c_{x}, x\right) \mathbb{B}^{d+1}\right)$. It holds that a compact-valued multifunction with closed graph is upper semicontinuous (see e.g. Theorem 1 p. 41 in [1]).

The notion of semiconcave function will also be used (see [10]):

Definition 2.6. Let $\Omega \subseteq \mathbb{R}^{d}$ be open, $\bar{\omega}:[0,+\infty[\rightarrow[0,+\infty$ [ be an upper semicontinuous nondecreasing function such that $\lim _{r \rightarrow 0^{+}} \bar{\omega}(r)=0$. We say that a function $f: \Omega \rightarrow \mathbb{R}$ is semiconcave of modulus $\bar{\omega}$ if the inequality

$$
\lambda f(x)+(1-\lambda) f(y)-f(\lambda x+(1-\lambda) y) \leq \lambda(1-\lambda) \bar{\omega}(\|x-y\|)\|x-y\|
$$

holds for every $x, y \in \Omega, \lambda \in[0,1]$ such that $\lambda x+(1-\lambda) y \in \Omega$. We call locally semiconcave a function which is semiconcave on each compact convex subsets of its domain.

This definition generalizes the classical notion of semiconcavity, which concerns moduli $\omega(\cdot)$ of the form $\omega(r)=c r$, for a suitable constant $c>0$. If this is the case, we say that $f$ is semiconcave with linear modulus and we call $c$ semiconcavity constant. A function $f$ is called semiconvex if $-f$ is semiconcave.

The following result gives characterization of semiconcavity with linear modulus (see [10]).

Proposition 2.7. Let $\Omega \subseteq \mathbb{R}^{d}$ be open and $f: \Omega \rightarrow \mathbb{R}$ be a function. Then the following statements are equivalent:

(1) $f$ is semiconcave with linear modulus and semiconcavity constant $c>0$;

(2) the function $x \mapsto f(x)-c|x|^{2}$ is concave in every convex subset of $\Omega$.

(3) $f \in C^{0}(\Omega)$ and $f(y+h)+f(y-h)-2 f(y) \leq c|h|^{2}$ for any $y, h \in \mathbb{R}^{d}$ such that the segment joining $y+h$ and $y-h$ is contained in $\Omega$.

We recall some basic concepts from geometric measure theory. The major references are [22], [24] and [2]. 
Definition 2.8. Let $\Omega \subseteq \mathbb{R}^{d}$ be open and $L \geq 0$. We say that a function $f: \Omega \rightarrow \mathbb{R}$ is Lipschitz continuous of rank $L$ in $\Omega$, and we will write $f \in \operatorname{Lip}(\Omega)$, if

$$
|f(x)-f(y)| \leq L\|x-y\| \quad \text { for all } x, y \in \Omega .
$$

We say that $f$ is locally Lipschitz continuous in $\Omega$, and we write $f \in \operatorname{Lip}_{\text {loc }}(\Omega)$, if for every open bounded set $U \subseteq \Omega$ we have $f \in \operatorname{Lip}(U)$.

Rademacher's theorem, see e.g. Theorem 2.14 in [2], states that if $f \in \operatorname{Lip}_{\text {loc }}(\Omega)$ then $f$ is differentiable at $\mathscr{L}^{d}$-a.e. point of $\Omega$.

Definition 2.9. Let $A \subseteq \mathbb{R}^{d}$ and $0 \leq p \leq d$. The $p$-dimensional Hausdorff measure $\mathscr{H}^{p}(A)$ is defined by $\mathscr{H}^{p}(A)=\lim _{\delta \rightarrow 0^{+}} \mathscr{H}_{\delta}^{p}(A)$, where

$$
\mathscr{H}_{\delta}^{p}(A)=\omega_{p} \inf \left\{\sum_{i=1}^{\infty}\left(\operatorname{diam}\left(U_{i}\right)\right)^{p}: A \subseteq \bigcup_{i} U_{i}, \operatorname{diam}\left(U_{i}\right)<\delta\right\},
$$

and

$$
\omega_{p}:=\frac{2^{p} \Gamma\left(\frac{p}{2}+1\right)}{\pi^{p / 2}}, \quad \quad \Gamma(p):=\int_{0}^{\infty} t^{p-1} e^{-t} d t .
$$

When $p \in \mathbb{N}$, the constant $\omega_{p}$ equals the $p$-dimensional Lebesgue measure of the unit ball in $\mathbb{R}^{p}$. Moreover, $\mathscr{H}^{d}(A)=\mathscr{L}^{d}(A)$ for any $A \subseteq \mathbb{R}^{d}$.

We define the Hausdorff dimension $\operatorname{dim}_{\mathscr{H}}(A)$ of $A$ by setting

$$
\operatorname{dim}_{\mathscr{H}}(A):=\inf \left\{p \geq 0: \mathscr{H}^{p}(A)=0\right\}=\sup \left\{p \geq 0: \mathscr{H}^{p}(A)=+\infty\right\} .
$$

Let $k \in \mathbb{N}$, we say that $A \subseteq \mathbb{R}^{d}$ is countably $\mathscr{H}^{k}$-rectifiable if

$$
A \subseteq \mathcal{N} \cup \bigcup_{i=1}^{\infty} S_{i}
$$

where $S_{i}$ are suitable $k$-dimensional Lipschitz surfaces ${ }^{1}$ and $\mathcal{N}$ is a $\mathscr{H}^{k}$-negligible set. We say that $A$ is $\mathscr{H}^{k}$-rectifiable if it is countably $\mathscr{H}^{k}$-rectifiable and $\mathscr{H}^{k}(A)<\infty$, while $A$ is locally $\mathscr{H}^{k}$-rectifiable if $A \cap K$ is $\mathscr{H}^{k}$-rectifiable for any compact set $K \subseteq \mathbb{R}^{d}$. Given an open subset $\Omega$ of $\mathbb{R}^{d}$ and a Lipschitz continuous function $f: \Omega \rightarrow \mathbb{R}^{m}$, with Lipschitz rank $L \geq 0$, for every $0 \leq k \leq d$, the estimate $\mathscr{H}^{k}(f(S)) \leq L^{k} \mathscr{H}^{k}(S)$ holds for all $S \subseteq \Omega$. (see Proposition 2.49(iv) in $[2])$.

We will use several times the following result about Hausdorff and Radon measures, for which we refer to [2, Theorem 2.56].

\footnotetext{
${ }^{1}$ We say that $S \subseteq \mathbb{R}^{d+1}$ is a $k$-dimensional Lipschitz surface if for any $x \in S$ there exists an open neighbourhood $U \ni x$, a $k$-dimensional plane $\pi$ and a Lipschitz function $g: \pi \rightarrow \pi^{\perp}$ such that

$$
S \cap U=\left\{(y, f(y)) \in \pi \times \pi^{\perp}: y \in \pi\right\} \cap U .
$$
}


Theorem 2.10. Let $\Omega \subseteq \mathbb{R}^{d}$ be an open set and $\mu$ a positive Radon measure in $\Omega$. Then for any $t \in] 0,+\infty[$ and any Borel set $B \subseteq \Omega$ the following implications hold:

$$
\begin{aligned}
& \limsup _{r \rightarrow 0^{+}} \frac{\mu\left(x+r \mathbb{B}^{d}\right)}{\omega_{p} r^{p}} \geq t \quad \forall x \in B \quad \Longrightarrow \quad \mu \geq t \mathscr{H}^{p}\llcorner B \\
& \underset{r \rightarrow 0^{+}}{\limsup } \frac{\mu\left(x+r \mathbb{B}^{d}\right)}{\omega_{p} r^{p}} \leq t \quad \forall x \in B \quad \Longrightarrow \quad \mu \leq 2^{p} t \mathscr{H}^{p}\llcorner B
\end{aligned}
$$

The concepts of functions of bounded variation and of sets with finite perimeter will also be used (see p. 117 and p. 143 in [2]).

Definition 2.11. Let $\Omega \subseteq \mathbb{R}^{d}$ be open, and $u \in L^{1}(\Omega)$. We say that $u$ is a function of bounded variation in $\Omega$ (denoted by $u \in B V(\Omega)$ ) if the distributional derivative of $u$ is representable by a finite Radon measure in $\Omega$, i.e., if

$$
\int_{\Omega} u \frac{\partial \varphi}{\partial x_{i}} d x=-\int_{\Omega} \varphi d D_{i} u \text { for all } \varphi \in C_{c}^{\infty}(\Omega), i=1, \ldots, d
$$

for some Radon measure $D u=\left(D_{1} u, \ldots, D_{d} u\right)$. We denote by $\|D u\|$ the total variation of the vector measure $D u$, i.e.,

$$
\|D u\|(\Omega):=\sup \left\{\int_{\Omega} u(x) \operatorname{div} \phi(x) d x: \phi \in C_{c}^{1}\left(\Omega, \mathbb{R}^{d}\right),\|\phi\|_{L^{\infty}(\Omega)} \leq 1\right\} .
$$

Accordingly, $u \in L_{\text {loc }}^{1}(\Omega)$ is a function of locally bounded variation in $\Omega$ (denoted by $u \in$ $\left.B V_{\text {loc }}(\Omega)\right)$ if $u \in B V(U)$ for every open set $U \subset \subset \Omega$.

Definition 2.12. Let $E \subseteq \mathbb{R}^{d+1}$ be $\mathscr{L}^{d+1}$-measurable, and let $\Omega \subseteq \mathbb{R}^{d+1}$ be open. $E$ has finite perimeter in $\Omega$ if its characteristic function $\chi_{E}$ has bounded variation in $\Omega$; the perimeter of $E$ in $\Omega$ is $P(E, \Omega):=\left\|D \chi_{E}\right\|(\Omega)$. We say that $E$ has locally finite perimeter in $\Omega$ if $P(E, U)<+\infty$ for every open set $U \subset \subset \Omega$.

Definition 2.13. Let $\mu$ be a Radon measure on $\mathbb{R}^{d}$, and let $M$ be the union of all open sets $U \subseteq \mathbb{R}^{d}$ such that $\mu(U)=0$; the complement of $M$ is called the support of $\mu$ and it is denoted by $\operatorname{supp}(\mu)$.

The following concept of normal vector was introduced by E. De Giorgi.

Definition 2.14. Let $\Omega$ be a nonempty open subset of $\mathbb{R}^{d+1}$ and $E \subseteq \mathbb{R}^{d+1}$ be a set of finite perimeter in $\Omega$; we call reduced boundary of $E$ in $\Omega$ the set $\partial^{*} E$ of all points $x \in \operatorname{supp}\left(\left\|D \chi_{E}\right\|\right) \cap \Omega$ such that

$$
\nu_{E}(x):=\lim _{\rho \rightarrow 0^{+}} \frac{D \chi_{E}\left(x+\rho \mathbb{B}^{d+1}\right)}{\left\|D \chi_{E}\right\|\left(x+\rho \mathbb{B}^{d+1}\right)}=\frac{d D \chi_{E}}{d\left\|D \chi_{E}\right\|}(x)
$$

exists in $\mathbb{R}^{d+1}$ and satisfies $\left\|\nu_{E}(x)\right\|=1$. The function $-\nu_{E}: \partial^{*} E \rightarrow \mathbb{R}^{d+1}$ is called the measure theoretic outer normal to $E$ in $x$.

Finally, the following measure-theoretic concepts will be used in our analysis. 
Definition 2.15. Let $E \subseteq \mathbb{R}^{d+1}$ be a Borel set. For $x \in \mathbb{R}^{d+1}$ and $0 \leq k \leq d+1$ we set

$$
\delta_{E}^{k}(x)=\lim _{\rho \rightarrow 0^{+}} \frac{\mathscr{H}^{k}\left(E \cap\left(x+\rho \mathbb{B}^{d+1}\right)\right)}{\omega_{k} \rho^{k}}
$$

provided the limit exists. It is well known that for $k=d+1$ the limit actually exists and is equal to 1 for $\mathscr{L}^{d+1}$-a.e. $x \in E$; we call Lebesgue point of $E$ any such point.

Definition 2.16. Let $E \subseteq \mathbb{R}^{d+1}$ be $\mathscr{L}^{d+1}$-measurable. We set (see p. 158 in [2])

$$
\begin{aligned}
E^{0} & :=\left\{x \in \mathbb{R}^{d+1}: \delta_{E}^{d+1}(x)=0\right\}, & & \text { the measure theoretic exterior of } E ; \\
E^{1} & :=\left\{x \in \mathbb{R}^{d+1}: \delta_{E}^{d+1}(x)=1\right\}, & & \text { the measure theoretic interior of } E ; \\
\partial_{M} E & :=\mathbb{R}^{d+1} \backslash\left(E^{0} \cup E^{1}\right), & & \text { the measure theoretic boundary of } E .
\end{aligned}
$$

Concerning the relations among the above introduced concepts of boundary, we recall the following (see Theorem 3.61, p. 158, in [2]).

Theorem 2.17 (De Giorgi, Federer). Let $\Omega$ be a nonempty open subset of $\mathbb{R}^{d+1}$ and $E \subseteq \mathbb{R}^{d+1}$ be a set of finite perimeter in $\Omega$. Then $\partial^{*} E \cap \Omega$ is $\mathscr{H}^{d}$-rectifiable,

$$
\begin{aligned}
& D \chi_{E}\left\llcorner\Omega=\nu_{E} \mathscr{H}^{d}\left\llcorner\left(\partial^{*} E \cap \Omega\right)\right.\right. \\
& \left\|D \chi_{E}\right\|\left\llcorner\Omega=\mathscr{H}^{d}\left\llcorner\left(\partial^{*} E \cap \Omega\right)\right.\right. \\
& \partial^{*} E \cap \Omega \subseteq\left\{x \in \Omega: \delta_{E}^{d+1}(x)=1 / 2\right\} \subseteq \partial_{M} E \cap \Omega \subseteq \partial E \cap \Omega
\end{aligned}
$$

and

$$
\mathscr{H}^{d}\left(\Omega \backslash\left(E^{0} \cup \partial^{*} E \cup E^{1}\right)\right)=0 .
$$

In particular, $E$ has density either 0 , or $\frac{1}{2}$, or 1 at $\mathscr{H}^{d}$-a.e. $x \in \Omega$, and $\mathscr{H}^{d}\left(\partial_{M} E \backslash \partial^{*} E\right)=0$.

We conclude this Section with a Lemma which will be used several times in the sequel; the interested reader is refered to [2, Section 3.2].

Lemma 2.18. Let $f \in B V(a, b)$; then there exists a measurable set $I \subseteq(a, b)$ such that $\mathscr{L}^{1}(I)=b-a$ and

$$
\|D f\|(a, b) \geq|f(t)-f(s)| \quad \text { for any } t, s \in I .
$$

\section{StANDING HYPOTHESIS AND FIRST CONSEQUENCES}

Definition 3.1. Let $U \subseteq \mathbb{R}^{d+1}$ be open and $K \subseteq \mathbb{R}^{d+1}$ be nonempty and relatively closed in $U$. We say that $K$ is $N$-regular in $U$ if there exists an upper semicontinuous multifunction $N: \partial K \cap U \rightrightarrows \mathbb{S}^{d}$ such that for every $x \in \partial K \cap U$ the following two properties hold:

$(\mathrm{N} 1) \emptyset \neq N(x) \subseteq N_{K}^{F}(x) \cap \mathbb{S}^{d}$

$(\mathrm{N} 2)$ there exist $\left.\delta_{x} \in\right] 0$, dist $(x, \partial U)\left[\right.$ and a continuous function $\omega_{x}: \mathbb{R}^{+} \rightarrow \mathbb{R}^{+}$with $\lim _{r \rightarrow 0^{+}} \omega_{x}(r) / r=0$ and satisfying the following uniformity property: for every $y_{1} \in$ $\left(x+\delta_{x} \mathbb{B}^{d+1}\right) \cap \partial K$ there exists $\nu\left(y_{1}\right) \in N\left(y_{1}\right)$ such that

$$
\left\langle\nu\left(y_{1}\right), y_{2}-y_{1}\right\rangle \leq \omega_{x}\left(\left\|y_{2}-y_{1}\right\|\right) \text { for all } y_{2} \in\left(x+\delta_{x} \mathbb{B}^{d+1}\right) \cap K .
$$

We will say that $K \subseteq \mathbb{R}^{d+1}$ is $N$-regular if $K$ is $N$-regular in $\mathbb{R}^{d+1}$. 
Remark 3.2. Roughly speaking, a set is $N$-regular if we can find a suitable selection of the normal cone satisfying good properties of uniformity and continuity. Clearly, every set $K$ that is the closure of an open $C^{1}$ domain is $N$-regular: just set $N(x):=\left\{\nu_{K}(x)\right\}$ for every $x \in \partial K$, $\nu_{K}(x)$ being the exterior unit normal to $K$.

Also, a closed convex set $C$ is $N$-regular with

$$
N(x)=N_{C}^{F}(x) \cap \mathbb{S}^{d}=\left\{v \in \mathbb{S}^{d}: v \in \mathbb{R}^{d+1}:\langle v, y-x\rangle \leq 0 \text { for all } y \in C\right\} .
$$

Remark 3.3. One could give several different characterizations of $N$-regular sets. For instance, $K$ is $N$-regular in $U$ if and only if one of the following condition holds:

(1) $K \cap \bar{V}$ is $N$-regular in $\mathbb{R}^{d+1}$ for any $C^{1}$ domain $V \subset \subset U$;

(2) $K \cap \bar{V}$ is $N$-regular in $\mathbb{R}^{d+1}$ for any $C^{1}$ domain with $\bar{V} \subseteq U$;

The same holds if one replaces the $C^{1}$ smoothness of $V$, in the previous conditions, with the assumption that $V$ is a $N$-regular domain.

Remark 3.4. Possibly replacing the set-valued map $N$ with $x \mapsto \overline{N(x)}$, when $K$ is $N$-regular in $U$ we can always assume that $N$ has closed graph.

Definition 3.5. Let $U \subseteq \mathbb{R}^{d+1}$ be open and $K \subseteq \mathbb{R}^{d+1}$ be nonempty and relatively closed in $U$; let also $z \in \partial K \cap U, \theta \in] 0,1]$ and $C \geq 0$. We define

$$
\mathscr{N}_{K}^{C, \theta, U}(z):=\left\{\zeta \in \mathbb{R}^{d+1}:\left\langle\zeta, z^{\prime}-z\right\rangle \leq C \cdot\|\zeta\| \cdot\left\|z^{\prime}-z\right\|^{1+\theta} \text { for all } z^{\prime} \in K \cap U\right\} .
$$

If $K$ is closed, $U=\mathbb{R}^{d+1}$ and $z \in \partial K$ we will simply write $\mathscr{N}_{K}^{C, \theta}(z)$ instead of $\mathscr{N}_{K}^{C, \theta, \mathbb{R}^{d+1}}(z)$.

We notice that $0 \in \mathscr{N}_{K}^{C, \theta, U}(z) \subseteq N_{K}^{F}(z)$. Moreover (we omit the trivial proofs)

(a) if $\zeta \in \mathscr{N}_{K}^{C, \theta, U}(x)$, then $\mu \zeta \in \mathscr{N}_{K}^{C, \theta, U}(x)$ for all $\mu \geq 0$;

(b) the multifunction $\mathscr{N}_{K}^{C, \theta, U}: \partial K \cap U \rightrightarrows \mathbb{R}^{d+1}$ has closed graph.

Let now $\Omega \subseteq \mathbb{R}^{d}$ be nonempty and open and $f: \Omega \rightarrow \mathbb{R}$ be upper semicontinuous. By adapting the previous definition, for $\left(x, \beta_{x}\right) \in \partial$ hypo $f \cap(\Omega \times \mathbb{R})$ we define $\hat{\mathscr{N}}_{\text {hypo } f}^{C, \theta}\left(x, \beta_{x}\right)$ as the set of those $(v, \lambda) \in \mathbb{R}^{d} \times \mathbb{R}$ such that

$$
\left\langle(v, \lambda),\left(y-x, \beta-\beta_{x}\right)\right\rangle \leq C\|(v, \lambda)\|\left(\|y-x\|^{1+\theta}+\left|\beta-\beta_{x}\right|^{1+\theta}\right) \quad \forall(y, \beta) \in \text { hypo } f .
$$

We notice that there exist constants $c_{1}, c_{2}>0$ depending only on $d$ and $\theta$ such that

$$
\mathscr{N}_{\text {hypo } f}^{c_{1} C, \theta, \Omega \times \mathbb{R}}\left(x, \beta_{x}\right) \subseteq \hat{\mathscr{N}}_{\text {hypo } f}^{C, \theta}\left(x, \beta_{x}\right) \subseteq \mathscr{N}_{\text {hypo } f}^{c_{2} C, \theta, \Omega \times \mathbb{R}}\left(x, \beta_{x}\right) .
$$

It is clear from the definition that also $\hat{\mathscr{N}}_{\text {hypo } f}^{c, \theta}: \partial$ hypo $f \cap(\Omega \times \mathbb{R}) \rightrightarrows \mathbb{R}^{d+1}$ has closed graph.

Example 3.6. Geometrically speaking, formula (7) expresses in a quantitative way the existence of a subquadratic surface touching the set $K$ from outside. Figure 2 gives example of these subquadratic surfaces "lying outside" the set $K$ (in a sense given by (7)) in the 2-dimensional case. We draw the curves implicitly defined by the equation

$$
\langle v, P-Q\rangle=\|v\| \cdot\|P-Q\|^{1+\theta},
$$

by taking $Q=0,\|v\|=1$ and different values of $\theta$. Notice that the case $\theta=1$ corresponds to a circle and, as $\theta \rightarrow 0$, the suface shrinks to its longest axis of symmetry, whose direction 
is given by $v$. The pictures show the situation for $v=(\cos \varphi, \sin \varphi)$ respectively in the cases $\varphi \in\{0, \pi / 6, \pi / 2\}$ and $\theta \in\{1,1 / 2,1 / 4,1 / 8\}$.
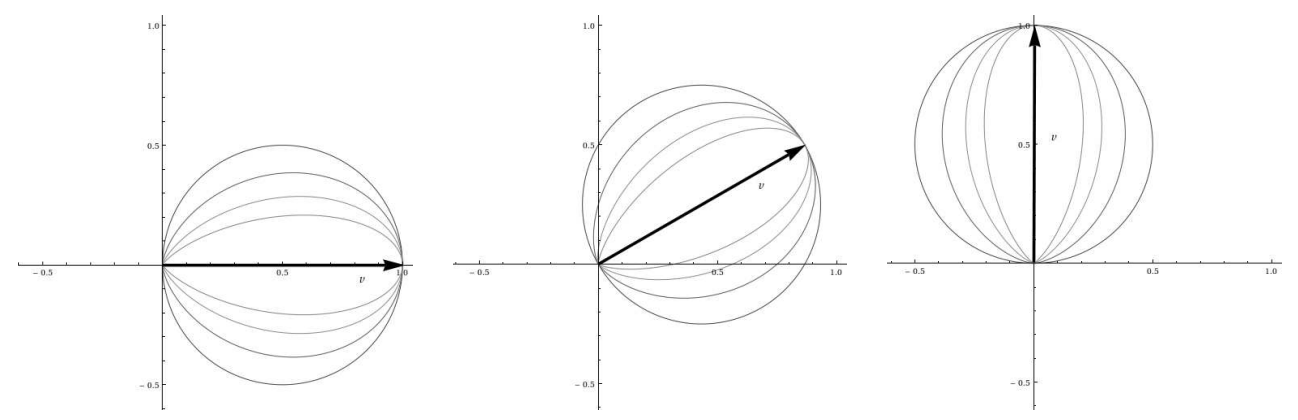

Figure 2. The subquadratic surfaces in $\mathbb{R}^{2}, \varphi=0, \pi / 6, \pi / 2$.

We are ready now to introduce the classes of sets and functions subject of our investigation.

Definition 3.7. Let $U \subseteq \mathbb{R}^{d+1}$ and $\Omega \subseteq \mathbb{R}^{d}$ be open. We define

$$
\begin{aligned}
& \mathscr{F}^{U}:=\{K \subseteq U: K \text { is relatively closed in } U \text { and } \exists C \geq 0,0<\theta \leq 1 \text { s.t. } \\
& \left.\mathscr{N}_{K}^{C, \theta, U}(z) \neq\{0\} \text { for all } z \in \partial K \cap U\right\} \\
& \mathscr{F}:=\mathscr{F}^{\mathbb{R}^{d+1}} \\
& \mathscr{F}(\Omega):=\left\{f: \Omega \rightarrow \mathbb{R}: f \text { u.s.c., hypo } f \in \mathscr{F}^{\Omega \times \mathbb{R}}\right\} \\
& =\{f: \Omega \rightarrow \mathbb{R}: f \text { u.s.c., } \exists C \geq 0,0<\theta \leq 1 \text { such that } \\
& \left.\hat{\mathscr{N}}_{\text {hypo } f}^{C, \theta}\left(x, \beta_{x}\right) \neq\{0\} \forall\left(x, \beta_{x}\right) \in \partial \text { hypo } f \cap(\Omega \times \mathbb{R})\right\} .
\end{aligned}
$$

Remark 3.8. One could be tempted to define the class $\mathscr{F}(\Omega)$ as that of those functions $f$ such that hypo $f \in \mathscr{F}$. Anyway, it is desirable for $\mathscr{F}(\Omega)$ to contain at least smooth functions, and one can check that (with this second definition) not even the constant functions would belong to $\mathscr{F}(\Omega)$ when $\partial \Omega$ is "very irregular".

If $K \in \mathscr{F}^{U}$, then there exist $C>0,0<\theta \leq 1$ such that $K$ is $N$-regular in $U$ with

$$
N(x):=\mathscr{N}_{K}^{C, \theta, U}(x) \cap \mathbb{S}^{d} \subseteq N_{K}^{F}(x), \quad \omega_{x}(r):=r^{1+\theta} \quad \forall x \in \partial K \cap U .
$$

The upper semicontinuity of $N$ follows from the fact that $\mathscr{N}_{K}^{C, \theta, U}(x)$ has closed graph.

Definition 3.9. Let $C>0$. We say that a closed set $K \in \mathscr{F}$

a. satisfies the uniform exterior sphere condition of radius $\frac{1}{2 C}$ if $\mathscr{N}_{K}^{C, 1}(z) \neq\{0\}$ for all $z \in \partial K$

b. has positive reach if $\mathscr{N}_{K}^{C, 1}(z)=N_{K}^{F}(z) \neq\{0\}$ for all $z \in \partial K$. In this case, we set

$$
\operatorname{reach}(K)=\inf \left\{\frac{1}{2 C}: \mathscr{N}_{K}^{C, 1}(z) \neq\{0\} \text { for all } z \in \partial K\right\} ;
$$

c. has locally positive reach if $K \cap r \mathbb{B}^{d+1}$ has positive reach for any $r>0$; 
d. is convex if $\operatorname{reach}(K)=+\infty$.

We refer the reader to $[21,24]$ for a survey of the properties satisfied by sets with positive reach, on which the class $\mathscr{F}$ is modeled.

\section{Regularity RESUlts FOR SETS: RECTIFIABILITy OF THE SINGUlaR SET AND FINITE PERIMETER}

In this section we will prove regularity results for the boundary of a closed set $K \subseteq \mathbb{R}^{d+1}$ in a quite general setting. They will be used later to prove fine regularity properties for functions in the class $\mathscr{F}(\Omega)$.

The first result extends an analogous result for the class of sets with positive reach proved by Federer in Remark 4.15 of [24]. It concerns rectifiability and Hausdorff dimension of the sets of points where the Fréchet normal cone has large dimension (i.e. corners or cusps): more precisely, if we partition the boundary of $K$ according to the dimension of the normal cone to the boundary, we have that the Hausdorff dimension of such sections decreases as the dimension of the normal cone increases. Roughly speaking, points with large normal cone are relatively few.

Definition 4.1. Let $K \subseteq \mathbb{R}^{d+1}$ be closed; for $j=1, \ldots, d+1$ we define

$$
\begin{aligned}
& K^{(j)}:=\left\{x \in \partial K: \operatorname{dim}\left(N_{\partial K}^{F}(x)\right) \geq j\right\}, \\
& K_{+}^{(j)}:=\left\{x \in \partial K: \operatorname{dim}\left(N_{K}^{F}(x)\right) \geq j\right\}, \\
& K_{-}^{(j)}:=\left\{x \in \partial K: \operatorname{dim}\left(N_{\overline{\mathbb{R}^{d+1} \backslash K}}^{F}(x)\right) \geq j\right\} .
\end{aligned}
$$

We notice that $K^{\left(j_{1}\right)} \supseteq K^{\left(j_{2}\right)}, K_{+}^{\left(j_{1}\right)} \supseteq K_{+}^{\left(j_{2}\right)}, K_{-}^{\left(j_{1}\right)} \supseteq K_{-}^{\left(j_{2}\right)}$ if $1 \leq j_{1} \leq j_{2} \leq d+1$, and that $K_{ \pm}^{(j)} \subseteq K^{(j)}$. Clearly, $K^{(1)}=\left\{x \in \partial K: N_{\partial K}^{F}(x) \neq\{0\}\right\}$.

In order to use local arguments, we will need the following estimate which gives some uniformity with respect to the elements of the normal cone:

Lemma 4.2. Let $K \subseteq \mathbb{R}^{d+1}$ be closed and define $\left.\left.\left.\left.\delta: K^{(1)} \times\right] 0,1\right] \rightarrow\right] 0,+\infty\right]$ by setting

$$
\begin{aligned}
& \delta(x, \varepsilon) \\
& \quad=\frac{1}{2} \sup \left\{\delta \in \mathbb{R}:\langle v, y-x\rangle \leq \varepsilon\|y-x\| \text { for all } y \in \partial K \cap\left(x+\delta \mathbb{B}^{d+1}\right), v \in N_{\partial K}^{F}(x) \cap \mathbb{S}^{d}\right\} .
\end{aligned}
$$

Then for every $x \in K^{(1)}$ and $0<\varepsilon \leq 1$ we have $\delta(x, \varepsilon)>0$.

Proof. Since $N_{\partial K}^{F}(x) \cap \mathbb{S}^{d}$ is compact, we can find a finite set $A_{x}:=\left\{v_{1}, \ldots, v_{N_{\varepsilon}}\right\} \subseteq N_{\partial K}^{F}(x) \cap \mathbb{S}^{d}$ such that

$$
N_{\partial K}^{F}(x) \cap \mathbb{S}^{d} \subseteq A_{x}+\frac{\varepsilon}{2} \mathbb{B}^{d+1} .
$$

By definition, there exist $\delta_{1}, \ldots, \delta_{N_{\varepsilon}}>0$ such that

$$
\left\langle v_{i}, y-x\right\rangle \leq \frac{\varepsilon}{2}\|y-x\| \quad \text { for every } y \in \partial K \cap\left(x+\delta_{i} \mathbb{B}^{d+1}\right), i=1, \ldots, N_{\varepsilon} .
$$


Set $\delta:=\min \left\{\delta_{i}: i=1, \ldots, N_{\varepsilon}\right\}>0$. For every $v \in N_{\partial K}^{F}(x) \cap \mathbb{S}^{d}$, there exists $i \in\left\{1,2, \ldots, N_{\varepsilon}\right\}$ such that $\left\|v-v_{i}\right\| \leq \frac{\varepsilon}{2}$. Hence, for every $y \in \partial K \cap\left(x+\delta \mathbb{B}^{d+1}\right)$, it holds

$$
\langle v, y-x\rangle=\left\langle v_{i}, y-x\right\rangle+\left\langle v-v_{i}, y-x\right\rangle \leq \varepsilon\|y-x\| .
$$

Thus $\delta(x, \varepsilon) \geq \delta / 2>0$ and the proof is concluded.

We are now ready to prove the first main result of the paper.

Proof of Theorem 1.1. We begin by constructing a countable covering $\left\{K_{n, m, h, l}^{(j)}\right\}_{n, m, h, l \in \mathbb{N}}$ of $K^{(j)}$; we will prove later (see Claim 3) that each element of the covering is rectifiable and this will establish our result.

Define the function $w:\left(\mathbb{R}^{d+1}\right)^{j} \rightarrow[0,1]$

$$
w\left(v_{1}, \ldots, v_{j}\right):=\min \left\{\left\|\sum_{i=1}^{j} \alpha_{i} v_{i}\right\|: \alpha_{i} \in \mathbb{R}, \sum_{i=1}^{j}\left|\alpha_{i}\right|=1\right\} .
$$

We notice that $w$ is continuous and invariant under permutations of its arguments. Roughly speaking, $w\left(v_{1}, \ldots, v_{j}\right)$ measures how much $V:=\left\{v_{1}, \ldots, v_{j}\right\}$ is far from being an orthonormal set, a case which occurs precisely when $w=1 / \sqrt{j}$. Moreover

$$
w\left(v_{1}, \ldots, v_{j}\right)=0 \Longleftrightarrow v_{1}, \ldots, v_{j} \text { are linearly dependent. }
$$

By symmetry, we will write $w(V)$ instead of $w\left(v_{1}, \ldots, v_{j}\right)$ if $V=\left\{v_{1}, \ldots, v_{j}\right\}$.

Consider the set

$$
\mathcal{A}^{(j)}:=\left\{V^{\prime} \subseteq \mathbb{Q}^{d+1}: \operatorname{card}\left(V^{\prime}\right)=\operatorname{dim} \operatorname{Span}\left(V^{\prime}\right)=j\right\},
$$

where $\operatorname{card}(X)$ denotes the number of the elements of a set $X$. Being $\mathcal{A}^{(j)}$ countable, we can order its elements and write $\mathcal{A}^{(j)}=\left\{V_{n}^{\prime}\right\}_{n \in \mathbb{N}}$.

We set $V_{n}^{(j)}=\operatorname{Span}\left(V_{n}^{\prime}\right)$ and consider the countable set of $j$-dimensional planes $\mathcal{V}^{(j)}:=$ $\left\{V_{n}^{(j)}\right\}_{n \in \mathbb{N}}$. Define also

$$
W_{n}^{(j)}:=\left(V_{n}^{(j)}\right)^{\perp} \quad \text { and } \quad \mathcal{W}^{(j)}:=\left\{W_{n}^{(j)}\right\}_{n \in \mathbb{N}}, \quad n \in \mathbb{N} .
$$

Let $\left\{a_{l}\right\}_{l \in \mathbb{N}}$ be a countable dense set in $\mathbb{R}^{d+1}$. Finally, for $x \in K^{(j)}$ choose $V_{x} \subseteq N_{\partial K}^{F}(x) \cap \mathbb{S}^{d}$ such that $V_{x}=\left\{v_{x}^{(1)}, \ldots, v_{x}^{(j)}\right\}$ and $\operatorname{dim} \operatorname{Span}\left(V_{x}\right)=j$.

Given $n, m, h, l \in \mathbb{N}$, let $v_{1}, \ldots, v_{j} \in \mathbb{Q}^{d+1}$ be such that $V_{n}^{\prime}=\left\{v_{1}, \ldots, v_{j}\right\}$ and set

$$
K_{n, m, h, l}^{(j)}:=\left\{x \in K^{(j)} \cap\left(a_{l}+\frac{1}{2(h+1)} \mathbb{B}^{d+1}\right): \begin{array}{l}
w\left(V_{x}\right) \geq \frac{1}{m+3}, \delta\left(x, \frac{1}{2(m+3)^{2}}\right) \geq \frac{1}{h+1}, \\
\left\|v_{x}^{(i)}-v_{i}\right\| \leq \frac{1}{2(m+3)^{2}} \text { for } i=1, \ldots, j
\end{array}\right\},
$$

where $\delta\left(x, \frac{1}{2(m+3)^{2}}\right)$ is as in Lemma 4.2 with $\varepsilon=\left(2(m+3)^{2}\right)^{-1}$.

Claim 1: $K^{(j)} \subseteq \bigcup_{n, m, h, l \in \mathbb{N}} K_{n, m, h, l}^{(j)}$. 
Proof of Claim 1: Let $x \in K^{(j)}$. Since $V_{x}$ is a set of linearly independent vectors, we have that $w\left(V_{x}\right)>0$, hence there exists $\bar{m} \in \mathbb{N}$, such that $w\left(V_{x}\right) \geq 1 /(m+3)$ for all $m \in \mathbb{N}$ with $m>\bar{m}$. By the density of $\mathbb{Q}$ in $\mathbb{R}$, for all $m \geq \bar{m}$ we can choose $V^{\prime}=\left\{v_{1}, \ldots, v_{j}\right\} \subseteq \mathbb{Q}^{d+1}$ such that

$$
\max _{i=1, \ldots, j}\left\|v_{i}-v_{x}^{(i)}\right\| \leq \frac{1}{2(m+3)^{2}} .
$$

For $m$ large enough, we have also that $\operatorname{dim} \operatorname{Span}\left(V^{\prime}\right)=j$ and

$$
w\left(V^{\prime}\right) \geq \frac{1}{2(m+3)},
$$

hence there exists $n \in \mathbb{N}$ such that $V^{\prime}=V_{n}^{\prime}$. According to Lemma 4.2 we have $\delta\left(x, \frac{1}{2(m+3)^{2}}\right)>0$, thus we can choose $h \in \mathbb{N}$ such that

$$
\delta\left(x, \frac{1}{2(m+3)^{2}}\right)>\frac{1}{h+1} .
$$

By the density of $\left\{a_{l}\right\}_{l \in \mathbb{N}}$ in $\mathbb{R}^{d+1}$ we can select $l \in \mathbb{N}$ such that $x \in a_{l}+\frac{1}{2(h+1)} \mathbb{B}^{d+1}$. This proves that $x \in K_{n, m, h, l}^{(j)}$ and Claim 1 is proved.

Claim 2: For any $x_{1}, x_{2} \in K_{n, m, h, l}^{(j)}$ the orthogonal projection $\pi_{W_{n}^{(j)}}: K_{n, m, h, l}^{(j)} \rightarrow W_{n}^{(j)}$ satisfies

$$
\left\|\pi_{W_{n}^{(j)}}\left(x_{2}-x_{1}\right)\right\|^{2} \geq \frac{m+1}{m+3}\left\|x_{2}-x_{1}\right\|^{2} .
$$

Proof of Claim 2: By assumption we have

$$
\begin{aligned}
\left\|x_{1}-x_{2}\right\| & \leq\left\|x_{1}-a_{l}\right\|+\left\|x_{2}-a_{l}\right\| \leq \frac{1}{h+1} \\
& \leq \min \left\{\delta\left(x_{1}, \frac{1}{2(m+3)^{2}}\right), \delta\left(x_{2}, \frac{1}{2(m+3)^{2}}\right)\right\} .
\end{aligned}
$$

Let $v_{1}, \ldots v_{j} \in \mathbb{Q}^{d+1}$ be such that $V_{n}^{\prime}=\left\{v_{1}, \ldots, v_{j}\right\}$; by the definition of $\delta$, for every $i=1, \ldots, j$ it holds

$$
\begin{aligned}
& \left\langle v_{i}, x_{2}-x_{1}\right\rangle \leq\left\langle v_{i}-v_{x_{1}}^{(i)}, x_{2}-x_{1}\right\rangle+\left\langle v_{x_{1}}^{(i)}, x_{2}-x_{1}\right\rangle \leq \frac{1}{(m+3)^{2}}\left\|x_{2}-x_{1}\right\|, \\
& \left\langle v_{i}, x_{1}-x_{2}\right\rangle \leq\left\langle v_{i}-v_{x_{2}}^{(i)}, x_{1}-x_{2}\right\rangle+\left\langle v_{x_{2}}^{(i)}, x_{1}-x_{2}\right\rangle \leq \frac{1}{(m+3)^{2}}\left\|x_{2}-x_{1}\right\|,
\end{aligned}
$$

and this gives

$$
\left|\left\langle v_{i}, x_{2}-x_{1}\right\rangle\right| \leq \frac{1}{(m+3)^{2}}\left\|x_{2}-x_{1}\right\| \quad \text { for every } i=1, \ldots, j .
$$

Given $v \in V_{n}^{(j)}, v \neq 0$, we can find (in a unique way) $\alpha_{i} \in \mathbb{R}, i=1, \ldots, j$ such that $v=$ $\sum_{i=1}^{j} \alpha_{i} v_{i} ;$ therefore

$$
\left|\left\langle\frac{v}{\|v\|}, x_{2}-x_{1}\right\rangle\right| \leq \frac{\sum_{i=1}^{j}\left|\alpha_{i}\right| \cdot\left|\left\langle v_{i}, x_{2}-x_{1}\right\rangle\right|}{\left\|\sum_{i=1}^{j} \alpha_{i} v_{i}\right\|} \leq \frac{\left\|x_{2}-x_{1}\right\|}{(m+3)^{2}} \frac{\sum_{i=1}^{j}\left|\alpha_{i}\right|}{\left\|\sum_{i=1}^{j} \alpha_{i} v_{i}\right\|} .
$$


Set $\beta_{i}:=\alpha_{i} / \sum_{s=1}^{j}\left|\alpha_{s}\right|$; we have $\sum_{i=1}^{j}\left|\beta_{i}\right|=1$ and thus

$$
\left|\left\langle\frac{v}{\|v\|}, x_{2}-x_{1}\right\rangle\right| \leq \frac{\left\|x_{2}-x_{1}\right\|}{(m+3)^{2}} \frac{1}{\left\|\sum_{i=1}^{j} \beta_{i} v_{i}\right\|} \leq \frac{\left\|x_{2}-x_{1}\right\|}{(m+3)^{2}} \frac{1}{w\left(v_{1}, \ldots, v_{j}\right)} \leq \frac{2}{m+3}\left\|x_{2}-x_{1}\right\|
$$

because $w\left(v_{1}, \ldots, v_{j}\right) \geq(2(m+3))^{-1}$ (recall (12)). Therefore,

$$
\begin{aligned}
\left\|\pi_{W_{n}^{(j)}}\left(x_{2}-x_{1}\right)\right\|^{2} & =\left\|x_{2}-x_{1}\right\|^{2}-\left\langle\pi_{V_{n}^{(j)}}\left(x_{2}-x_{1}\right), x_{2}-x_{1}\right\rangle \\
& \geq\left\|x_{2}-x_{1}\right\|^{2}-\frac{2}{m+3}\left\|\pi_{V_{n}^{(j)}}\left(x_{2}-x_{1}\right)\right\|\left\|x_{2}-x_{1}\right\| \\
& \geq \frac{m+1}{m+3}\left\|x_{2}-x_{1}\right\|^{2} .
\end{aligned}
$$

This proves Claim 2.

Claim 3: The set $K_{n, m, h, l}^{(j)}$ is $\mathscr{H}^{d-j+1}$-rectifiable.

Proof of Claim 3: By (13), for each $n, m, h, l$ the inverse map

$$
\pi_{W_{n}^{(j)}}^{-1}: \pi_{W_{n}^{(j)}}\left(K_{n, m, h, l}^{(j)}\right) \rightarrow K_{n, m, h, l}^{(j)} .
$$

is Lipschitz continuous and, by Kirszbraun's Theorem, it can be extended to a Lipschitz function defined on the whole $W_{n}^{(j)}$. This establishes the Claim.

The theorem is now an easy consequence of Claims 1 and 3.

Corollary 4.3. Let $K \subseteq \mathbb{R}^{d+1}$ be closed and $N$-regular. Then $\partial K \cap U$ is a finite union of Lipschitz graphs for any open set $U \subset \subset \mathbb{R}^{d+1}$ and, in particular, $K$ has locally finite perimeter in $\mathbb{R}^{d+1}$.

Proof. By $N$-regularity we have $\partial K=K^{(1)}$; moreover, for every $x \in \partial K$ there exist $0<\delta_{x}<1$ and $\omega_{x}:\left[0,+\infty\left[\rightarrow\left[0,+\infty\left[\right.\right.\right.\right.$ such that $\lim _{r \rightarrow 0^{+}} \omega_{x}(r) / r=0$ and the following holds. For every $y_{1}, y_{2} \in\left(x+\delta_{x} \mathbb{B}^{d+1}\right) \cap \partial K$ there exist $\nu\left(y_{1}\right) \in N\left(y_{1}\right), \nu\left(y_{2}\right) \in N\left(y_{2}\right)$ such that

$$
\left\langle\nu\left(y_{1}\right), y_{2}-y_{1}\right\rangle \leq \omega_{x}\left(\left\|y_{1}-y_{2}\right\|\right) \quad \text { and } \quad\left\langle\nu\left(y_{2}\right), y_{1}-y_{2}\right\rangle \leq \omega_{x}\left(\left\|y_{1}-y_{2}\right\|\right) .
$$

Let $U \subseteq \mathbb{R}^{d+1}$ be a bounded open set; by compactness we can find a finite set $\left\{x_{l}: l=\right.$ $0, \ldots, L\} \subseteq \partial K$ such that

$$
\partial K \cap \bar{U} \subseteq \bigcup_{l=1}^{L} x_{l}+\delta_{x_{l}} \mathbb{B}^{d+1} .
$$

By $N$-regularity, for each $l=1, \ldots, L$ we can find $0<\delta_{l}^{\prime}<1$ such that for every $y \in \partial K \cap$ $\left(x_{l}+\delta_{x_{l}} \mathbb{B}^{d+1}\right)$ there exists $v_{y} \in N(y)$ with

$$
\left\langle v_{y}, z-y\right\rangle \leq \frac{1}{3}\|z-y\| \quad \text { for any } z \in \partial K \cap\left(x_{l}+\delta_{x_{l}} \mathbb{B}^{d+1}\right) \text { with }\|z-y\| \leq \delta_{l}^{\prime} .
$$

By compactness of $\partial K \cap\left(x_{l}+\delta_{x_{l}} \overline{\mathbb{B}^{d+1}}\right)$, we can select a finite set $\left\{y_{1}, \ldots, y_{M_{1}}\right\} \subseteq \partial K$ such that

$$
\partial K \cap \bar{U} \subseteq \bigcup_{l=1}^{L} \partial K \cap\left(x_{l}+\delta_{x_{l}} \overline{\mathbb{B}^{d+1}}\right) \subseteq \bigcup_{l=1}^{L} \bigcup_{h=1}^{M_{1}} \partial K \cap\left(y_{h}+\frac{\delta_{l}^{\prime}}{2} \mathbb{B}^{d+1}\right) \cap\left(x_{l}+\delta_{x_{l}} \overline{\mathbb{B}^{d+1}}\right) .
$$


We set $B_{l, h}:=\left(y_{h}+\frac{\delta_{l}^{\prime}}{2} \mathbb{B}^{d+1}\right) \cap\left(x_{l}+\delta_{x_{l}} \overline{\mathbb{B}^{d+1}}\right)$, and we notice that if $y \in B_{l, h} \cap \partial K$, then there exists $v_{y} \in N(y)$ such that

$$
\left\langle v_{y}, z-y\right\rangle \leq \frac{1}{3}\|z-y\| \quad \text { for every } z \in B_{l, h} \cap \partial K .
$$

Now, by compactness of $\mathbb{S}^{d}$, we can find $M_{2} \in \mathbb{N}$ and a finite set $\left\{v_{1}, \ldots, v_{M_{2}}\right\} \subseteq \mathbb{S}^{d}$ such that

$$
\mathbb{S}^{d} \subseteq \bigcup_{i=1}^{M_{2}}\left(v_{i}+\mathbb{B}^{d+1} / 3\right)
$$

For $m=1, \ldots, M_{2}$ and $h=0, \ldots, M_{1}$ consider the set

$$
K_{l, h, m}:=\left\{y \in B_{l, h} \cap \partial K:\left\|v_{y}-v_{m}\right\| \leq \frac{1}{3}\right\} .
$$

We have that $\bigcup_{l, h, m} K_{l, h, m} \supseteq U \cap \partial K$. Given $y_{1}, y_{2} \in K_{l, h, m}$, we have

$$
\begin{aligned}
& \left\langle v_{m}, y_{1}-y_{2}\right\rangle=\left\langle v_{m}-v_{y_{2}}, y_{1}-y_{2}\right\rangle+\left\langle v_{y_{2}}, y_{1}-y_{2}\right\rangle \leq \frac{2}{3}\left\|y_{1}-y_{2}\right\|, \\
& \left\langle v_{m}, y_{2}-y_{1}\right\rangle=\left\langle v_{m}-v_{y_{1}}, y_{2}-y_{1}\right\rangle+\left\langle v_{y_{1}}, y_{2}-y_{1}\right\rangle \leq \frac{2}{3}\left\|y_{1}-y_{2}\right\|,
\end{aligned}
$$

whence $\left|\left\langle v_{m}, y_{2}-y_{1}\right\rangle\right| \leq \frac{2}{3}\left\|y_{1}-y_{2}\right\|$ and thus, as in the proof of Theorem 1.1,

$$
\left\|\pi_{v_{m}^{\perp}}\left(y_{1}\right)-\pi_{v_{m}^{\perp}}\left(y_{2}\right)\right\|^{2}=\left\|\pi_{v_{m}^{\perp}}\left(y_{1}-y_{2}\right)\right\|^{2}=\left\|y_{1}-y_{2}\right\|^{2}-\left\langle v_{m}, y_{2}-y_{1}\right\rangle^{2} \geq \frac{5}{9}\left\|y_{1}-y_{2}\right\|^{2} .
$$

So $\pi_{v_{m}^{\perp}}$ is (linear) injective and hence invertible on $K_{l, h, m}$. We denote by $f_{m}:=\pi_{v_{m}^{\perp}}^{-1}$ its inverse map, which is Lipschitz continuous (with Lipschitz constant not greater than $3 / \sqrt{5}$ ) and is defined on a subset of a $d$-dimensional space. We can extend it to a map defined on the whole of $v_{m}^{\perp}$.

We notice that by Lipschitz continuity of $f_{m}$ we have:

$$
\mathscr{H}^{d}\left(f_{m}\left(\pi_{v_{m}^{\perp}}\left(B_{l, h}\right)\right)\right) \leq(3 / \sqrt{5})^{d} \mathscr{H}^{d}\left(\mathbb{B}^{d}\right) .
$$

Then

$$
\mathscr{H}^{d}(U \cap \partial K) \leq \mathscr{H}^{d}\left(\bigcup_{l, h, m} f_{m}\left(\pi_{v_{m}^{\perp}}\left(B_{l, h}\right)\right)\right) \leq L \cdot M_{1} \cdot M_{2} \cdot(3 / \sqrt{5})^{d} \mathscr{H}^{d}\left(\mathbb{B}^{d}\right)<+\infty .
$$

According to Theorem 4.5.11 and Remark 4.5.12 pp. 506-508 in [25], we have that $P(K, U)<$ $+\infty$ (see also Theorem $\mathrm{E}$ in [12], recalling that $\partial_{M} K \subseteq \partial K$ ). The proof is concluded by the arbitrarity of $U$.

Remark 4.4. Let us notice that Theorem 1.1 holds for any $K \subseteq \mathbb{R}^{d+1}$ : the closedness assumption has never been used. Clearly, Definition 4.1 can be stated for general $K$. Theorem 1.1 implies that if at each point $x$ of the boundary of a closed set $K$ there exists at least one nontrivial Fréchet normal (either external to the set, i.e. in $N_{K}^{F}(x)$, or internal to the set, i.e. in $N_{\mathbb{R}^{d+1} \backslash K}^{F}(x)$ ), then the boundary $\partial K$ is countably $\mathscr{H}^{d}$-rectifiable. To prove Corollary 4.3, i.e., that the set has locally finite perimeter, it is crucial to strengthen the hypotheses of Theorem 1.1 by assuming the $N$-regularity of the set. 
For example, it is easy to see that the hypograph $K \subseteq \mathbb{R}^{2}$ of the function $u: \mathbb{R} \rightarrow \mathbb{R}$

$$
u(x):= \begin{cases}x^{2} \sin \frac{1}{x^{2}} & \text { if } x \neq 0 \\ 0 & \text { if } x=0\end{cases}
$$

which is closed because $u$ is continuous, satisfies $K^{(1)}=\partial K$ (because $u$ is differentiable) but its perimeter measure is not locally finite. This happens because $K$ is not $N$-regular in any neighbourhood of $(0,0)$, while it is of class $C^{1,1}$ away from the origin.

The proof of Corollary 4.3 can be easily adapted to prove its "local" version.

Corollary 4.5. Let $U \subseteq \mathbb{R}^{d+1}$ be open and let $K \subseteq \mathbb{R}^{d+1}$ be relativey closed and $N$-regular in $U$. Then, for any open set $V \subset \subset U$ we have that $\partial K \cap V$ is a finite union of Lipschitz graphs; in particular, $K$ has locally finite perimeter in $U$.

The application of these results to sets in the class $\mathscr{F}^{U}$ is immediate.

Corollary 4.6. Let $U \subseteq \mathbb{R}^{d+1}$ be open and let $K \in \mathscr{F}^{U}$. Then

(1) $\partial K \cap U=K^{(1)} \cap U$ and $\partial K \cap V$ is a finite union of Lipschitz graphs for any $V \subset \subset U$;

(2) for any $j=1, \ldots, d, K^{(j)} \cap U$ is countably $\mathscr{H}^{d-j+1}$-rectifiable;

(3) $K$ has locally finite perimeter in $U$.

Proof. According to Definition 3.7, we have that $K$ is $N$-regular in $U$ and, in particular, $N_{K}^{F}(x) \neq\{0\}$ for all $x \in \partial K \cap U$. Thus $\partial K \cap U=K^{(1)} \cap U$ and the conclusion follows from Theorem 1.1 and Corollary 4.5.

Remark 4.7. Our result is strictly related to Theorem 5.8 in [4], where the authors estimate the perimeter of sets enjoying an internal cone property. Indeed, the same arguments of Corollary 4.3 (for $\theta=0,0<C<1$ ) easily gives the same conclusion of [4]. See also [26, Proposition 2.4].

\section{Application to Functions: $B V$ Regularity and Structure of Singular set}

In this section we will apply the results obtained in the previous one to closed sets that can be written as hypographs of upper semicontinuous functions possessing at least one normal direction at a.e. point of the boundary of their hypograph; our goal is to obtain regularity results for such functions.

Assume for simplicity that $f \in \mathscr{F}(\Omega) \cap C^{0}(\Omega)$. According to the second part of Corollary 4.6, we already know that at $\mathscr{L}^{d}$-a.e. point $x \in \Omega$ there exists an unique (up to the sign) unit Fréchet normal $(\zeta, \xi) \in \mathbb{R}^{d} \times \mathbb{R}$ to hypo $f$ at $(x, f(x))$. This is a necessary condition for $f$ to be differentiable at $x$, however it is not sufficient: in fact, if $\xi=0$, i.e. the (unique) unit normal to the hypograph is horizontal, then $\partial^{F} f(x)$ is empty, hence the function cannot be differentiable at $x$. For example, the graph of $f(x)=\operatorname{sign}(x) \sqrt{|x|}$ from $\mathbb{R}$ to $\mathbb{R}$ is of class $C^{1,1}$ but $\partial^{F} f(0)=\partial_{F} f(0)=\emptyset$, so the function cannot be differentiable at 0 .

Motivated by the previous considerations, we distinguish between three kinds of singularities that can occur:

(1) points $x$ where the Fréchet normal cone to the hypograph at $(x, \beta) \in \partial$ hypo $f$ reduces to $\{0\}$; 
(2) points $x$ where the Fréchet normal cone to the hypograph at $(x, \beta) \in$ Ohypo $f$ has dimension greater than 1 (e.g. corners, cusps...);

(3) points $x$ where the Fréchet normal cone to the hypograph at $(x, \beta) \in$ dhypo $f$ has dimension 1, but its unique (up to the sign) element of norm 1 is horizontal.

The first type of singularity is excluded by the definition of the class $\mathscr{F}(\Omega)$, while the second kind of singularity can be controlled thanks to Corollary 4.6. The third one is not yet covered by previous results.

In [5] it is proved that given a l.s.c. function $\left.\left.f: \mathbb{R}^{d} \rightarrow\right]-\infty,+\infty\right]$, the set of points where the lower Dini subdifferential contains more than element is $\mathscr{H}^{d-1}$-rectifiable. This result was later improved in [38], where it was proved that the set of points where the lower Dini subdifferential has convex dimension $k$ is $\mathscr{H}^{d-k}$-rectifiable. These results cannot deal with the third kind of singularities, since in that case the subdifferential is empty. Our purpose is to cover also this situation.

Definition 5.1. Let $\Omega$ be a nonempty open subset of $\mathbb{R}^{d}$ and $f: \Omega \rightarrow \mathbb{R}$ be a function. For each $x \in \Omega$, we define

$$
\begin{aligned}
& J_{f}:=\{x \in \Omega: \widetilde{f}(x) \neq \underset{\sim}{f}(x)\}=\{x \in \Omega: f \text { is not continuous at } x\}, \\
& S_{f}:=\left\{x \in \Omega \backslash J_{f}:\left(\mathbb{S}^{d-1} \times\{0\}\right) \cap N_{\text {hypo } f}^{F}(x, f(x)) \neq \emptyset\right\}, \\
& \mathscr{S}_{f}:=J_{f} \cup S_{f} .
\end{aligned}
$$

We begin with a trivial corollary of Theorem 1.1, dealing with the singularities corresponding to large dimension of the normal cone. Let us point out once more that for upper semicontinuous functions it holds $\underset{\sim}{f}=\underline{f} \leq \bar{f} \leq f=\tilde{f}$.

Corollary 5.2. Let $\Omega$ be a nonempty open subset of $\mathbb{R}^{d}$ and $f: \Omega \rightarrow \mathbb{R}$ be an upper semicontinuous function. Set $K=$ hypo $f$ and assume that

$$
N_{K}^{F}(x, \beta) \neq\{0\} \quad \text { for } \mathscr{H}^{d} \text {-a.e. }(x, \beta) \in \partial K \cap(\Omega \times \mathbb{R}) .
$$

Then for $\mathscr{L}^{d}$-almost every $x \in \Omega$ there exists $\zeta_{x} \in \mathbb{S}^{d}$ such that

$$
N_{K}^{F}(x, \beta) \subseteq \mathbb{R} \zeta_{x} \quad \text { for all } \beta \text { with }(x, \beta) \in \partial K \cap(\Omega \times \mathbb{R}) .
$$

Proof. Recalling Definition 4.1, we have that

$$
\mathscr{H}^{d}\left(\left(\partial K \backslash K^{(1)}\right) \cap(\Omega \times \mathbb{R})\right)=0 .
$$

By Theorem 1.1 and Remark $4.4, K^{(2)}$ is $\mathscr{H}^{d-1}$-rectifiable and hence $\mathscr{H}^{d}$-negligible. If $\pi$ : $\Omega \times \mathbb{R} \rightarrow \Omega$ denotes the canonical projection on $\Omega$, then $\Omega \cap\left(\pi\left(\partial K \backslash K^{(1)}\right) \cup \pi\left(K^{(2)}\right)\right)$ is $\mathscr{L}^{d}$-negligible, hence

$$
E:=\Omega \backslash\left(\pi\left(\partial K \backslash K^{(1)}\right) \cup \pi\left(K^{(2)}\right)\right)
$$

has the same measure of $\Omega$. It is enough to prove the statement for any point $x \in E$.

If $x \in E$, then by Lemma $2.4(x, \beta) \in \partial K$ if and only if $f(x) \leq \beta \leq f(x)$. By definition of $E$ we have $(x, \beta) \in K^{(1)} \backslash K^{(2)}$ for any such $\beta$, hence

$$
N_{K}^{F}(x, \beta) \subseteq \mathbb{R} \zeta_{x, \beta}
$$


for a suitable $\zeta_{x, \beta} \in \mathbb{S}^{d} \cap N_{K}^{F}(x, \beta)$. Hence it is enough to show that actually one can choose $\zeta_{x, \beta}=\zeta_{x, \underline{f}(x)}$, so that $\zeta_{x, \beta}$ is independent of $\beta$. This follows from Lemma $2.4(\mathrm{v})$, which gives

$$
\zeta_{x, \underline{f}(x)} \in N_{K}^{F}(x, \underline{f}(x)) \subseteq N_{K}^{F}(x, \beta) \subseteq \mathbb{R} \zeta_{x, \beta}
$$

for any $\beta \in[\underline{f}(x), f(x)]$.

One of our primary goals is to estimate the size of the singular set $\mathscr{S}_{f}$; to this aim it will be important to assume that $f$ is of class $B V$. We can now prove the second main result of the paper.

Proof of Theorem 1.2. We reason by contradiction and prove that the assumption $\mathscr{L}^{d}\left(\mathscr{S}_{f}\right)>0$ contradicts the fact that $f \in B V_{l o c}(\Omega)$. The first step consists in reducing the problem to estimate the total variation around points where the unit Fréchet normal to the hypograph is unique and horizontal. More precisely we define the set $T$ of points where the normal cone has dimension 1 and is horizontal

$$
T:=\left\{\left(x, \beta_{x}\right) \in \partial K \cap(\Omega \times \mathbb{R}): \text { there exists } v_{x} \in \mathbb{S}^{d-1} \text { with } N_{K}^{F}\left(x, \beta_{x}\right) \subseteq \mathbb{R}\left(v_{x}, 0\right)\right\},
$$

where as usual we have set $K:=$ hypo $f$. The projection of $T$ on the first $d$ components is the set

$$
S:=\left\{x \in \mathscr{S}_{f}: \exists v_{x} \in \mathbb{S}^{d-1}, \beta_{x} \in[\underline{f}(x), f(x)] \text { s.t. } N_{K}^{F}\left(x, \beta_{x}\right) \subseteq \mathbb{R}\left(v_{x}, 0\right)\right\} .
$$

Throughout this proof, for each $z \in S$ we denote by $\beta_{z}$ a real number such that

$$
\beta_{z} \in[\underline{f}(z), f(z)], \quad \operatorname{dim} N_{K}^{F}\left(z, \beta_{z}\right)=1, \quad \text { and } \quad N_{K}^{F}\left(z, \beta_{z}\right) \subseteq \mathbb{R}^{d} \times\{0\} .
$$

Claim 1: $\mathscr{L}^{d}\left(\mathscr{S}_{f} \backslash S\right)=0$.

Proof of Claim 1: Define (see also Definition 4.1)

$$
\mathcal{N}:=\pi\left(K_{+}^{(2)} \cap(\Omega \times \mathbb{R})\right) \cup\left\{x \in \Omega: \exists \beta_{x} \in \mathbb{R} \text { with }\left(x, \beta_{x}\right) \in \partial K \text { and } N_{K}^{F}\left(x, \beta_{x}\right)=\{0\}\right\},
$$

where $K_{+}^{(2)}$ is as in Definition 4.1 and $\pi$ is the canonical projection $\pi: \Omega \times \mathbb{R} \rightarrow \Omega$. By assumption, and using Theorem 1.1, Remark 4.4 and the Lipschitz continuity of $\pi$, we have that $\mathscr{L}^{d}(\mathcal{N})=0$. We notice that

a. if $x \in J_{f} \backslash \mathcal{N}$ then (by Lemma 2.4) there exists $\underline{f}(x)<\beta_{x}<f(x)$ such that

$$
N_{K}^{F}\left(x, \beta_{x}\right) \subseteq \mathbb{R}^{d} \times\{0\} \quad \text { and } \operatorname{dim} N_{K}^{F}\left(x, \beta_{x}\right)=1,
$$

hence $x \in S$.

b. by definition, $S \supseteq S_{f} \backslash \mathcal{N}$.

This gives $S \supseteq \mathscr{S}_{f} \backslash \mathcal{N}$, i.e., $\mathscr{S}_{f} \backslash S \subseteq \mathcal{N}$, and Claim 1 is proved.

By Claim 1, for our purposes it will suffice to show that $\mathscr{L}^{d}(S)=0$.

Claim 2: There exists $c=c(d)>0$ such that for every $\varepsilon \in] 0,1 / 16\left[\right.$ and for $\mathscr{L}^{d}$-a.e. $x \in S$ there exists $\delta_{x, \varepsilon}>0$ such that

$$
\left.\|D f\|\left(x+\delta \mathbb{B}^{d}\right) \geq \frac{c}{\varepsilon} \delta^{d} \quad \forall \delta \in\right] 0, \delta_{x, \varepsilon}[.
$$


Proof of Claim 2: Let $\varepsilon \in] 0,1 / 16[$ be fixed. By compactness, there exist $m \in \mathbb{N}$ and $v_{1}, \ldots, v_{m} \in \mathbb{S}^{d-1}$ such that

$$
\mathbb{S}^{d-1} \subseteq \bigcup_{i=1}^{m}\left(v_{i}+\mathbb{B}^{d} / 8\right) .
$$

Given $i \in\{1, \ldots, m\}$ and $\delta>0$ we define

$$
S^{i}:=\left\{x \in S: \operatorname{dist}\left(\left(v_{i}, 0\right), N_{K}^{F}\left(x, \beta_{x}\right)\right)<1 / 8\right\}
$$

and

$$
\begin{gathered}
S_{0}^{i, \delta}:=\left\{x \in S^{i}: \operatorname{Sq}(x, 4 \delta) \subseteq \Omega \text { and } \exists v_{x} \in v_{i}+\mathbb{B}^{d} / 4 \text { with }\left(v_{x}, 0\right) \in N_{K}^{F}\left(x, \beta_{x}\right)\right. \text { and } \\
\left.\left\langle v_{x}, y-x\right\rangle \leq \varepsilon\left(\|y-x\|+\left|f(y)-\beta_{x}\right|\right) \forall y \in \operatorname{Sq}(x, 4 \delta)\right\} .
\end{gathered}
$$

Clearly, $S^{i}=\bigcup_{\delta>0} S_{0}^{i, \delta}$. Denote by $S^{i, \delta} \subseteq S_{0}^{i, \delta}$ the set of Lebesgue points of $S_{0}^{i, \delta}$; since

$$
\begin{aligned}
& S^{i, \delta_{1}} \subseteq S^{i, \delta_{2}} \text { for any } 0<\delta_{2}<\delta_{1} \\
& \mathscr{L}^{d}\left(S^{i, \delta}\right)=\mathscr{L}^{d}\left(S_{0}^{i, \delta}\right) \rightarrow \mathscr{L}^{d}\left(S^{i}\right) \text { monotonically increasing as } \delta \rightarrow 0^{+},
\end{aligned}
$$

we have

$$
\mathscr{L}^{d}\left(S^{i} \backslash \bigcup_{\delta>0} S^{i, \delta}\right)=0
$$

and thus

$$
\mathscr{L}^{d}\left(S \backslash \bigcup_{i, \delta} S^{i, \delta}\right)=0 .
$$

Therefore it is enough to prove the claim for any point $x \in S$ for which there exist $i, \delta_{0}$ such that $x \in S^{i, \delta_{0}}$.

Let then $x \in S^{i, \delta_{0}}$ and $\left.\delta \in\right] 0, \delta_{1}$ [ be fixed; here, $\delta_{1}=\delta_{1}(x)<\delta_{0}$ is a positive constant which will be chosen later. For any $y \in S^{i, \delta} \cap \mathrm{Sq}(x, \delta)$ we have $y=x+u+t v_{i}$ for suitable $u \in v_{i}^{\perp} \cap \mathrm{Sq}(0, \delta)$ and $\left.t \in\right]-\delta, \delta\left[\right.$. By assumption there exists $v_{y} \in v_{i}+\mathbb{B}^{d} / 8$ such that

$$
\left(v_{y}, 0\right) \in N_{K}^{F}\left(y, \beta_{y}\right) \quad \text { and } \quad\left\langle v_{y}, z-y\right\rangle \leq \varepsilon\left(\|z-y\|+\left|f(z)-\beta_{y}\right|\right) \forall z \in \operatorname{Sq}(y, 4 \delta) .
$$

In particular, for any $z$ such that $z=x+u+s v_{i}$ for $\left.s \in\right] 2 \delta$, $3 \delta\left[\right.$ (i.e., $z-y=(s-t) v_{i}$ ) we have

$$
z \in \operatorname{Sq}(y, 4 \delta) \text { and } \delta<s-t=\|z-y\|<4 \delta .
$$

Thus, for any $\beta \leq f(z)$ we have

$$
\begin{aligned}
\delta \leq\left\langle v_{i}, z-y\right\rangle & =\left\langle v_{i}-v_{y}, z-y\right\rangle+\left\langle v_{y}, z-y\right\rangle \\
& \leq\left\langle v_{i}-v_{y},(s-t) v_{i}\right\rangle+\varepsilon\left(|s-t|+\left|\beta-\beta_{y}\right|\right) \\
& <\frac{1}{2} \delta+\varepsilon\left(4 \delta+\left|\beta-\beta_{y}\right|\right) \\
& <\frac{3}{4} \delta+\varepsilon\left|\beta-\beta_{y}\right| .
\end{aligned}
$$

Therefore

$$
\varepsilon\left|\beta-\beta_{y}\right| \geq \frac{1}{4} \delta \quad \forall \beta \leq f(z) .
$$


If $\beta_{y} \leq f(z)$, then we are allowed to take $\beta=\beta_{y}$ in the previous inequality obtaining $\delta \leq 0$, a contradiction. So we must have $\beta_{y}>f(z)$ and taking $\beta=f(z)$ in (14) we get

$$
f(y)-f(z) \geq \beta_{y}-f(z) \geq \frac{\delta}{4 \varepsilon}
$$

because $f(y)=\tilde{f}(y) \geq \beta_{y} \geq \underline{f}(y)$ by upper semicontinuity.

Since $x$ is a Lebesgue point for $S^{i, \delta_{0}}$ for any $\left.\delta \in\right] 0, \delta_{0}\left[\right.$, there exists a positive $\delta_{1}<\delta_{0}$ such that for all $\delta \in] 0, \delta_{1}[$

$$
\mathscr{L}^{d}\left(S^{i, \delta} \cap \mathrm{Sq}(x, \delta)\right) \geq \mathscr{L}^{d}\left(S^{i, \delta_{0}} \cap \mathrm{Sq}(x, \delta)\right) \geq \frac{1}{2} \mathscr{L}^{d}(\mathrm{Sq}(x, \delta))=2^{d-1} \delta^{d} .
$$

For any $u \in v_{i}^{\perp} \cap \operatorname{Sq}(0, \delta)$ denote by $L_{u} \subseteq \operatorname{Sq}(x, \delta)$ the line segment joining $x+u-\delta v_{i}$ and $x+u+\delta v_{i}$. Moreover, define $\left.f^{u}:\right]-4 \delta, 4 \delta[\rightarrow \mathbb{R}$ by

By Fubini's theorem

$$
f^{u}(r):=f\left(x+u+r v_{i}\right) .
$$

$$
\mathscr{L}^{d}\left(S^{i, \delta} \cap \mathrm{Sq}(x, \delta)\right)=\int_{v_{i}^{\perp} \cap \mathrm{Sq}(0, \delta)} \mathscr{L}^{1}\left(S^{i, \delta} \cap L_{u}\right) d \mathscr{L}^{d-1}(u),
$$

and, since the integrand is not greater than $2 \delta$, we must have

$$
\mathscr{L}^{d-1}\left(\left\{u \in v_{i}^{\perp} \cap \operatorname{Sq}(0, \delta): \mathscr{L}^{1}\left(S^{i, \delta} \cap L_{u}\right)>0\right\}\right) \geq 2^{d-2} \delta^{d-1},
$$

otherwise (16) would be contradicted.

It is well known that $f^{u} \in B V_{l o c}(-4 \delta, 4 \delta)$ for $\mathscr{L}^{d-1}$-a.e. $u$, hence the set

$$
U^{x, i, \delta}:=\left\{u \in v_{i}^{\perp} \cap \mathrm{Sq}(0, \delta): \mathscr{L}^{1}\left(S^{i, \delta} \cap L_{u}\right)>0 \text { and } f^{u} \in B V_{l o c}(-4 \delta, 4 \delta)\right\}
$$

satisfies $\mathscr{L}^{d-1}\left(U^{x, i, \delta}\right) \geq 2^{d-2} \delta^{d-1}$. By Lemma 2.18 , for any $u \in U^{x, i, \delta}$ there exists $\left.I^{u} \subseteq\right]-4 \delta, 4 \delta[$ such that $]-4 \delta, 4 \delta\left[\backslash I^{u}\right.$ is $\mathscr{L}^{1}$-negligible and

$$
\left\|D f^{u}\right\|(-4 \delta, 4 \delta) \geq\left|f^{u}(t)-f^{u}(s)\right| \quad \forall t, s \in I^{u} .
$$

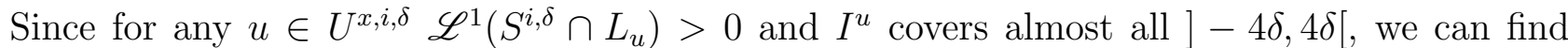
$\left.t \in I^{u} \cap\right]-\delta, \delta\left[\right.$ such that $y:=x+u+t v_{i} \in S^{i, \delta} \cap L_{u}$; moreover we can choose $\left.s \in I^{u} \cap\right] 2 \delta, 3 \delta[$. The previous inequality and (15) give

$$
\left\|D f^{u}\right\|(-4 \delta, 4 \delta) \geq f^{u}(t)-f^{u}(s)=f(y)-f(z) \geq \frac{\delta}{4 \varepsilon} \quad \forall u \in U^{x, i, \delta}
$$

whence (see [2])

$$
\begin{aligned}
\|D f\|(\operatorname{Sq}(x, 4 \delta)) & \geq\left\|D_{v_{i}} f\right\|(\operatorname{Sq}(x, 4 \delta))=\int_{v_{i}^{\perp} \operatorname{Sq}(0,4 \delta)}\left\|D f^{u}\right\|(-4 \delta, 4 \delta) d \mathscr{L}^{d-1} \\
& \geq \int_{U^{x, i, \delta}}\left\|D f^{u}\right\|(-4 \delta, 4 \delta) d \mathscr{L}^{d-1} \\
& \left.\geq \frac{\delta}{4 \varepsilon} \mathscr{L}^{d-1}\left(U^{x, i, \delta}\right) \geq \frac{2^{d-4}}{\varepsilon} \delta^{d} \quad \forall \delta \in\right] 0, \delta_{1}[,
\end{aligned}
$$

where we have denoted by $D_{v_{i}} f$ the distributional derivative of $f$ in direction $v_{i}$ and by $\left\|D_{v_{i}} f\right\|$ the total variation of such measure. This proves the Claim up to standard considerations. $\diamond$ 
Claim 2 allows to conclude easily. Let $\varepsilon \in] 0,1 / 16$ [ be fixed; Claim 2 implies that there exists $S^{\prime} \subseteq S$ with $\mathscr{L}^{d}\left(S \backslash S^{\prime}\right)=0$ such that

$$
\limsup _{\delta \rightarrow 0^{+}} \frac{\|D f\|\left(x+\delta \mathbb{B}^{d}\right)}{\omega_{d} \delta^{d}} \geq \frac{\tilde{c}}{\varepsilon} \quad \forall x \in S^{\prime}
$$

for a suitable $\tilde{c}=\tilde{c}(d)>0$. By Theorem 2.10 we deduce

$$
\|D f\|\left\llcorner S \geq\|D f\|\left\llcorner S^{\prime} \geq \frac{\tilde{c}}{\varepsilon} \mathscr{L}^{d}\left\llcorner S^{\prime}=\frac{\tilde{c}}{\varepsilon} \mathscr{L}^{d}\llcorner S .\right.\right.\right.
$$

In particular, for any $U \subset \subset \Omega$ we have

$$
\mathscr{L}^{d}(S \cap U) \leq \varepsilon \frac{\|D f\|(U)}{\tilde{c}}<+\infty
$$

which gives $\mathscr{L}^{d}(S \cap U)=0$ for any $U \subset \subset \Omega$. This proves the Theorem.

\section{Smoothness of FUnCtions With $N$-Regular Hypograph}

\subsection{Semiconcavity with modulus.}

We are going to study the regularity properties of upper semicontinuous functions $f$ such that hypo $f$ is $N$-regular. More precisely, we will prove that the set $\mathscr{S}_{f}$ introduced in Definition 5.1 is closed and $\mathscr{L}^{d}$-negligible. In particular, we will show that $f$ is locally semiconcave with a modulus $\omega$ in $\Omega \backslash \mathscr{S}_{f}$ and hence enjoys several regularity properties (see [10] or Chapter 10 in $[42])$.

The natural counterpart of Corollaries 4.3 and 4.5 for functions is given by the following

Proposition 6.1. Let $\Omega \subseteq \mathbb{R}^{d}$ be a nonempty open set and $f: \Omega \rightarrow \mathbb{R}$ be an upper semicontinuous function with $f \in L_{\mathrm{loc}}^{\infty}(\Omega)$. Assume that the closed set $K:=$ hypo $f$ is $N$-regular in $\Omega \times \mathbb{R}$. Then $f \in B V_{\text {loc }}(\Omega)$.

Proof. Let us prove that $f \in B V(U)$ for any open set $U$ such that $U \subset \subset \Omega$. By assumption there exists $M>0$ such that $|f(x)| \leq M$ for $\mathscr{L}^{d}$-a.e. $x \in \bar{U}$; this implies that

$$
P(K, U \times \mathbb{R})=P(K, U \times]-2 M, 2 M[)<\infty,
$$

where we have also used Corollary 4.5 , which guarantees that $K$ has locally finite perimeter in $\Omega \times \mathbb{R}$. This implies (see e.g. [28] or [27, Theorem 14.6]) that $f \in B V(U)$.

Remark 6.2. The assumption $f \in L_{l o c}^{\infty}(\Omega)$ is crucial in Proposition 6.1. Indeed, the hypograph of the u.s.c. function $f: \mathbb{R} \rightarrow \mathbb{R}$

$$
f(x)=-1 /|x| \text { if } x \neq 0, \quad f(0)=0
$$

is $N$-regular but $f \notin B V_{l o c}(\mathbb{R})$.

The following corollary is a consequence of Proposition 6.1 and Theorem 1.2.

Corollary 6.3. Under the assumption of Proposition 6.1, we have $\mathscr{L}^{d}\left(\mathscr{S}_{f}\right)=0$. 
We are now going to study the closure of the set $\mathscr{S}_{f}$ under the the $N$-regularity assumption on hypo $f$. Let us begin with the case in which $f$ is continuous.

Lemma 6.4. Let $\Omega \subseteq \mathbb{R}^{d}$ be open and $f: \Omega \rightarrow \mathbb{R}$ continuous. Assume that $K:=$ hypo $f$ is $N$-regular in $\Omega \times \mathbb{R}$. Let $x \in \Omega$ be such that $N_{K}^{F}(x, f(x)) \cap\left(\mathbb{S}^{d-1} \times\{0\}\right) \neq \emptyset$. Then, also $N(x, f(x)) \cap\left(\mathbb{S}^{d-1} \times\{0\}\right)$ is nonempty and, in particular, $\mathscr{S}_{f}=S_{f}$ is closed.

Proof. The proof is in spirit of Lemma 4.2 in [30]. Let $x \in \Omega, v \in \mathbb{S}^{d-1}$ be such that

$$
(v, 0) \in N_{K}^{F}(x, f(x)) .
$$

Set $x_{n}=x+v / n$. According to Clarke's Density Theorem (see Theorem 1.3.1 in [14]), for each $n \in \mathbb{N}$ there exists $\left\{z_{n}\right\}_{n \in \mathbb{N}} \subseteq \Omega$ such that

$$
\partial_{F} f\left(z_{n}\right) \neq \emptyset \text { and }\left\|z_{n}-x_{n}\right\|<1 / n^{2} .
$$

Since $N_{K}^{F}\left(z_{n}, f\left(z_{n}\right)\right) \neq\{0\}$ by the $N$-regularity property, we have that $f$ is differentiable at $z_{n}$. Moreover

$$
\frac{\left(-\nabla f\left(z_{n}\right), 1\right)}{\left\|\left(-\nabla f\left(z_{n}\right), 1\right)\right\|} \in N\left(z_{n}, f\left(z_{n}\right)\right) .
$$

Up to subsequence, still denoted by $\left\{z_{n}\right\}_{n \in \mathbb{N}}$, we may assume that the left hand side converges for $n \rightarrow+\infty$ to a vector $(\zeta, \xi) \in \mathbb{S}^{d}$. In order to prove the lemma, it is enough to show that

$$
\lim _{n \rightarrow+\infty}\left\|\nabla f\left(z_{n}\right)\right\|=+\infty
$$

which would give $\xi=0$ and $(\zeta, 0) \in N(x, f(x))$ because $N$ has closed graph.

Assume by contradiction that $\liminf _{n \rightarrow+\infty}\left\|\nabla f\left(z_{n}\right)\right\|=L \in \mathbb{R}$. Up to subsequence, still denoted by $\left\{z_{n}\right\}_{n \in \mathbb{N}}$ we may assume that $\left\{\nabla f\left(z_{n}\right)\right\}_{n \in \mathbb{N}}$ converges to some vector in $\mathbb{R}^{d}$. Recalling that

$$
(v, 0) \in N(x, f(x)) \text { and } \frac{\left(-\nabla f\left(z_{n}\right), 1\right)}{\left\|\left(-\nabla f\left(z_{n}\right), 1\right)\right\|} \in N\left(z_{n}, f\left(z_{n}\right)\right),
$$

we have for $n$ large enough

$$
\begin{aligned}
\left\langle v, z_{n}-x\right\rangle & \leq \omega_{x}\left(\sqrt{\left\|z_{n}-x\right\|^{2}+\left|\beta_{n}-f(x)\right|^{2}}\right), \\
\left\langle\frac{\left(-\nabla f\left(z_{n}\right), 1\right)}{\left\|\left(-\nabla f\left(z_{n}\right), 1\right)\right\|},\left(x-z_{n}, \beta-f\left(z_{n}\right)\right)\right\rangle & \leq \omega_{x}\left(\sqrt{\left\|x-z_{n}\right\|^{2}+\left|\beta-f\left(z_{n}\right)\right|^{2}}\right),
\end{aligned}
$$

for all $\beta \leq f(x), \beta_{n} \leq f\left(z_{n}\right)$ such that $|f(x)-\beta|,\left|\beta_{n}-f\left(z_{n}\right)\right|$ are sufficiently small.

Since $z_{n}-x=\left(v+n\left(z_{n}-x_{n}\right)\right) / n$, we have that $\frac{z_{n}-x}{\left\|z_{n}-x\right\|} \rightarrow v$. We take $\beta_{n}=f\left(z_{n}\right)$ in (17), divide by $\left\|z_{n}-x\right\|$ and pass to the $\lim$ inf as $n \rightarrow \infty$, obtaining

$$
1 \leq \liminf _{n \rightarrow+\infty} \frac{\omega_{x}\left(\sqrt{\left\|z_{n}-x\right\|^{2}+\left|f(x)-f\left(z_{n}\right)\right|^{2}}\right)}{\sqrt{\left\|z_{n}-x\right\|^{2}+\left|f(x)-f\left(z_{n}\right)\right|^{2}}} \cdot \sqrt{1+\left(\frac{\left|f(x)-f\left(z_{n}\right)\right|}{\left\|z_{n}-x\right\|}\right)^{2}} .
$$

This implies that

$$
\lim _{n \rightarrow \infty} \frac{\left|f(x)-f\left(z_{n}\right)\right|}{\left\|z_{n}-x\right\|}=+\infty
$$


otherwise the right hand side would vanish.

Now we distinguish two cases. If there exists a subsequence $\left\{z_{n_{k}}\right\}_{k \in \mathbb{N}} \subseteq\left\{z_{n}\right\}_{n \in \mathbb{N}}$ such that $f(x) \leq f\left(z_{n_{k}}\right)$, we take $\beta_{n_{k}}=f(x)$ in (17) and divide by $\left\|z_{n_{k}}-x\right\|$. We have

$$
\left\langle v, \frac{z_{n_{k}}-x}{\left\|z_{n_{k}}-x\right\|}\right\rangle \leq \frac{\omega_{x}\left(\left\|z_{n_{k}}-x\right\|\right)}{\left\|z_{n_{k}}-x\right\|} .
$$

On passing to the limit as $n_{k} \rightarrow+\infty$, the right hand side converges to 1 , while the left hand side vanishes leading to a contradiction.

Otherwise, there exists $n_{0}>0$ such that $f(x) \geq f\left(z_{n}\right)$ for all $n>n_{0}$ and by (19) we have

$$
\lim _{n \rightarrow \infty} \frac{f(x)-f\left(z_{n}\right)}{\left\|z_{n}-x\right\|}=+\infty .
$$

Using the fact that $\left\|\nabla f\left(z_{n}\right)\right\|$ is bounded, we take $\beta=f(x)$ in (18) and for $n$ sufficiently large we get

$$
\left\langle\frac{-\nabla f\left(z_{n}\right)}{\sqrt{1+\left|\nabla f\left(z_{n}\right)\right|^{2}}}, x-z_{n}\right\rangle+\frac{f(x)-f\left(z_{n}\right)}{\sqrt{1+\left|\nabla f\left(z_{n}\right)\right|^{2}}} \leq \omega_{x}\left(\sqrt{\left\|z_{n}-x\right\|^{2}+\left|f(x)-f\left(z_{n}\right)\right|^{2}}\right),
$$

whence

$$
\frac{f(x)-f\left(z_{n}\right)}{\left\|x-z_{n}\right\|} \leq \frac{\sqrt{L^{2}+1}}{\left\|x-z_{n}\right\|} \omega_{x}\left(\sqrt{\left\|z_{n}-x\right\|^{2}+\left|f(x)-f\left(z_{n}\right)\right|^{2}}\right)+L .
$$

Thus

$$
\frac{\frac{f(x)-f\left(z_{n}\right)}{\left\|x-z_{n}\right\|}-L}{\sqrt{1+\left(\frac{f(x)-f\left(z_{n}\right)}{\left\|z_{n}-x\right\|}\right)^{2}}} \leq \sqrt{L^{2}+1} \frac{\omega_{x}\left(\sqrt{\left\|z_{n}-x\right\|^{2}+\left|f(x)-f\left(z_{n}\right)\right|^{2}}\right)}{\sqrt{\left\|z_{n}-x\right\|^{2}+\left|f(x)-f\left(z_{n}\right)\right|^{2}}} .
$$

and, by (20), the left hand side tends to 1 , while the right hand side vanishes, leading to a contradiction.

We have thus proved that $\left\|\nabla f\left(z_{n}\right)\right\|$ is not bounded and this concludes the proof.

We now weaken the regularity hypothesis on $f$ by requiring it to be only upper semicontinuity.

Proposition 6.5. Let $\Omega$ be a nonempty open subset of $\mathbb{R}^{d}$ and $f: \Omega \rightarrow \mathbb{R}$ be an upper semicontinuous function. Assume that $K:=$ hypo $f$ is $N$-regular in $\Omega \times \mathbb{R}$. Then $\mathscr{S}_{f}$ is closed in $\Omega$.

Proof. Fix $x \in \Omega \backslash \mathscr{S}_{f}$. We need to prove that there exists $r_{x}>0$ such that $x+r_{x} \mathbb{B}^{d} \subseteq \Omega \backslash \mathscr{S}_{f}$. Assume by contradiction that for every $\varepsilon>0$ it holds

$$
\left(x+\varepsilon \mathbb{B}^{d}\right) \cap \mathscr{S}_{f} \neq \emptyset .
$$

Recalling that $\mathscr{S}_{f}=J_{f} \cup S_{f}$, two cases can occur.

Assume that for every $\varepsilon>0$ we have $\left(x+\varepsilon \mathbb{B}^{d}\right) \cap J_{f} \neq \emptyset$. Then we can take a sequence $\left\{x_{n}\right\}_{n \in \mathbb{N}} \subseteq J_{f}, x_{n} \rightarrow x$ such that for any $n$ there exists $\left.\beta_{n} \in\right] \underline{f}\left(x_{n}\right), f\left(x_{n}\right)[$. By statement (iv) in Lemma 2.4 we have

$$
\{0\} \neq N\left(x_{n}, \beta_{n}\right) \subseteq N_{K}^{F}\left(x_{n}, \beta_{n}\right) \subseteq \mathbb{R}^{d} \times\{0\},
$$


hence there exists $\left\{v_{n}\right\}_{n \in \mathbb{N}} \subseteq \mathbb{S}^{d-1}$ such that $\left(v_{n}, 0\right) \in N\left(x_{n}, \beta_{n}\right)$. Up to subsequences, we can assume that $\left(v_{n}, 0\right) \rightarrow\left(v_{x}, 0\right) \in \mathbb{S}^{d-1} \times\{0\}$. Since $x \in \Omega \backslash \mathscr{S}_{f}, f$ is continuous at $x$, hence $\beta_{n} \rightarrow f(x)$. It follows that $\left(v_{x}, 0\right) \in N(x, f(x)) \subseteq N_{K}^{F}(x, f(x))$ because $N$ has closed graph. This implies that $x \in S_{f}$ and contradicts the fact that $x \notin \mathscr{S}_{f} \supseteq S_{f}$.

Otherwise, there exists $\delta>0$ such that $\left(x+\delta \mathbb{B}^{d}\right) \cap J_{f}=\emptyset$, hence $f$ is continuous in $x+\delta \mathbb{B}^{d}$. By $(21)$, for all $\varepsilon \in] 0, \delta\left[\right.$ one has $\left(x+\varepsilon \mathbb{B}^{d}\right) \cap S_{f} \neq \emptyset$, hence there exist sequences $\left\{x_{n}\right\}_{n \in \mathbb{N}} \subseteq S_{f}$ and $\left\{v_{n}\right\}_{n \in \mathbb{N}} \subseteq \mathbb{S}^{d-1}$ such that

$$
\left(v_{n}, 0\right) \in N_{K}^{F}\left(x_{n}, f\left(x_{n}\right)\right) \text { and } x_{n} \rightarrow x .
$$

According to Lemma 6.4, we can assume that $\left(v_{n}, 0\right) \in N\left(x_{n}, f\left(x_{n}\right)\right)$. Since $N$ has closed graph, up to subsequence we have

$$
\left(v_{n}, 0\right) \rightarrow\left(v_{x}, 0\right) \in N(x, f(x)) \subseteq N_{K}^{F}(x, f(x))
$$

and thus $x \in S_{f}$, which gives again a contradiction. This concludes the proof.

This result extends a similar result proved in [30] for the exterior sphere case:

Theorem 6.6. Let $\Omega$ be a nonempty open subset of $\mathbb{R}^{d}$ and $f: \Omega \rightarrow \mathbb{R}$ be an upper semicontinuous function. Assume that $K:=$ hypo $f$ is $N$-regular in $\Omega \times \mathbb{R}$. Then $f$ is locally semiconcave with a modulus in the open set $\Omega \backslash \mathscr{S}_{f}$.

Proof. The set $\Omega \backslash \mathscr{S}_{f}$ is open by Proposition 6.5 and $f$ is continuous on $\Omega \backslash \mathscr{S}_{f}$. Let $x \in \Omega \backslash \mathscr{S}_{f}$; we begin by proving that $f$ is Lipschitz continuous in $x+\bar{\delta}_{x} \mathbb{B}^{d}$ for some $\bar{\delta}_{x}>0$ such that $x+\bar{\delta}_{x} \mathbb{B}^{d} \subseteq \Omega \backslash \mathscr{S}_{f}$. Since $K$ is $N$-regular and $f$ is continuous in a neighbourhood of $x$, there exists $\delta_{x}>0$ such that, for every $y \in x+\delta_{x} \mathbb{B}^{d}$, there exists $\left(-v_{y}, 1\right) \in N_{K}^{F}(y, f(y))$ such that

$$
\left\langle\frac{\left(-v_{y}, 1\right)}{\left\|\left(-v_{y}, 1\right)\right\|},(z-y, \beta-f(y))\right\rangle \leq \omega_{(x, f(x))}(\|(z-y, \beta-f(y))\|)
$$

for all $z \in \Omega$ and $\beta \leq f(z)$ sufficiently close to $x, f(x)$ respectively. Since $x \notin \mathscr{S}_{f}$, there are constants $0<\delta_{x}^{1} \leq \delta_{x}$ and $C>0$ such that $\left\|v_{y}\right\| \leq C$ for all $y \in x+\delta_{x}^{1} \mathbb{B}^{d}$; otherwise, we would have

$$
N(x, f(x)) \cap\left(\mathbb{S}^{d-1} \times\{0\}\right) \neq \emptyset
$$

and thus $x \in S_{f}$, a contradiction. Hence, by the continuity of $f$ on $x+\delta_{x} \mathbb{B}^{d}$, there exists a modulus $\omega_{x}:\left[0,2 \delta_{x}^{1}\right) \rightarrow[0,+\infty)$ such that $\lim _{r \rightarrow 0^{+}} \omega_{x}(r)=0$ and

$$
\left\langle-v_{y}, z-y\right\rangle+f(z)-f(y) \leq \omega_{x}(\|z-y\|)[\|z-y\|+|f(z)-f(y)|] \quad \forall z, y \in x+\delta_{x}^{1} \mathbb{B}^{d} .
$$

Given $y_{1}, y_{2} \in x+\delta_{x}^{1} \mathbb{B}^{d}$ we can assume without loss of generality that $f\left(y_{2}\right)>f\left(y_{1}\right)$ and the previous inequality can be rewritten as

$$
\left|f\left(y_{2}\right)-f\left(y_{1}\right)\right|\left(1-\omega_{x}\left(\left\|y_{2}-y_{1}\right\|\right)\right) \leq\left[\omega_{x}\left(\left\|y_{2}-y_{1}\right\|\right)+C\right]\left\|y_{2}-y_{1}\right\| .
$$

Since $\lim _{r \rightarrow 0^{+}} \omega_{x}(r)=0$ there exists $\bar{\delta}_{x}>0$ such that $f$ is Lipschitz continuous in $x+\bar{\delta}_{x} \mathbb{B}^{d}$ with Lipschitz constant $2 C$.

Using again (22), for any $w \in x+\bar{\delta}_{x} \mathbb{B}^{d}$ there exists $v_{w} \in \partial^{F} f(w)$ such that

$$
-\left\langle v_{w}, w^{\prime}-w\right\rangle+f\left(w^{\prime}\right)-f(w) \leq \omega_{x}^{1}\left(\left\|w^{\prime}-w\right\|\right)\left\|w^{\prime}-w\right\| \quad \forall w^{\prime} \in x+\bar{\delta}_{x} \mathbb{B}^{d},
$$


where $\omega_{x}^{1}=(1+2 C) \omega_{x}$. Let then $y, z \in x+\bar{\delta}_{x} \mathbb{B}^{d}$ and $t \in[0,1]$ be fixed; we can substitute $w^{\prime}=z$ (respectively, $w^{\prime}=y$ ) and $w=t y+(1-t) z$ in the previous inequality to get

$$
\begin{aligned}
& -t\left\langle v_{w}, z-y\right\rangle+f(z)-f(t y+(1-t) z) \leq \omega_{x}^{1}(t\|(z-y)\|) t\|z-y\| \\
& (1-t)\left\langle v_{w}, z-y\right\rangle+f(y)-f(t y+(1-t) z) \leq \omega_{x}^{1}((1-t)\|(z-y)\|)(1-t)\|z-y\| .
\end{aligned}
$$

Multiplying the first inequality by $(1-t)$, the second one by $t$ and summing up we obtain

$$
t f(y)+(1-t) f(z)-f(t y+(1-t) z) \leq t(1-t) \bar{\omega}_{x}(\|y-z\|)\|y-z\|
$$

where

$$
\bar{\omega}_{x}(r):=\max _{t \in[0,1]}\left\{\omega_{x}^{1}(t r)+\omega_{x}^{1}((1-t) r)\right\} .
$$

Thus $f$ is semiconcave with modulus $\bar{\omega}_{x}$ in $x+\bar{\delta}_{x} \mathbb{B}^{d}$. The proof is completed by observing that, if $U$ is an open set with $U \subset \subset \Omega \backslash \mathscr{S}_{f}$, then $U$ can be covered by finitely many balls $\left\{x_{i}+\right.$ $\left.\bar{\delta}_{x_{i}} \mathbb{B}^{d}\right\}_{i=1, \ldots, M}$ and the semiconcavity inequality is satisfied with modulus $\bar{\omega}_{U}(r)=\sum_{i=1}^{M} \bar{\omega}_{x_{i}}(r)$.

Corollary 6.3, Theorem 1.1 (together with Remark 4.4) and the differentiability properties of locally semiconcave functions (see e.g. [10]) allow us to summarize the regularity properties of functions belonging to $\mathscr{F}(\Omega) \cap L_{\text {loc }}^{\infty}$ in the following

Proposition 6.7. Assume that $f: \Omega \rightarrow \mathbb{R}$ satisfies the assumptions in Theorem 6.6 and $f \in L_{\text {loc }}^{\infty}(\Omega)$. Then

(1) $f$ is differentiable on the open set $\Omega \backslash \mathscr{S}_{f}$ out of countably $\mathscr{H}^{d-1}$-rectifiable set. Moreover

$$
\left\{x \in \Omega \backslash \mathscr{S}_{f}: f \text { is differentiable at } x\right\}=\left\{x \in \Omega \backslash \mathscr{S}_{f}: \# \partial^{F} f(x)=1\right\}
$$

and $\nabla f$ is continuous on its domain of definition.

(2) $f$ is differentiable $\mathscr{L}^{d}$-a.e. in $\Omega$.

(3) (Rifford) the set $\left\{x \in \Omega\right.$ : $\left.\operatorname{dim}\left(\operatorname{span} \partial^{F} f(x)\right) \geq k\right\}$ is countably $\mathscr{H}^{d-k+1}$-rectifiable.

\subsection{Reduced boundary and measure theoretic normal to $N$-regular hypographs.}

Since $N$-regular sets have (locally) finite perimeter, it is natural to investigate the properties of their reduced boundary and of the measure theoretic normal.

Proposition 6.8. Let $U$ be a nonempty open subset of $\mathbb{R}^{d+1}$ and let $K \subseteq \mathbb{R}^{d+1}$ be $N$-regular in $U$; let $x \in U$. Then $x \in \partial^{*} K$ if and only if $N_{K}^{F}(x) \cap \mathbb{S}^{d}$ contains a unique element; in this case one has

$$
N_{K}^{F}(x) \cap \mathbb{S}^{d}=\left\{-\nu_{K}(x)\right\} .
$$

Proof. By Corollary 4.5, $K$ has locally finite perimeter in $U$. Without loss of generality we may assume that $x=0$.

Claim 1: If $N_{K}^{F}(0) \cap \mathbb{S}^{d}$ contains more than one element, then $0 \notin \partial^{*} K$.

Proof of Claim 1. Assume that $N_{K}^{F}(0) \cap \mathbb{S}^{d}$ contains two different elements $v_{1}, v_{2}$. This implies that for every $\varepsilon>0$ there exists $\bar{\rho}>0$ such that

$$
\left.\begin{array}{l}
\left\langle v_{1}, y\right\rangle \leq \varepsilon \rho \\
\left\langle v_{2}, y\right\rangle \leq \varepsilon \rho
\end{array} \quad \forall y \in K \cap \rho \mathbb{B}^{d+1}, \rho \in\right] 0, \bar{\rho}[.
$$


In particular

$$
\left.K \cap \rho \mathbb{B}^{d+1} \subseteq\left(v_{1}^{0} \cap v_{2}^{0} \cap \rho \mathbb{B}^{d+1}\right)+2 \varepsilon \rho \mathbb{B}^{d+1} \quad \forall \rho \in\right] 0, \bar{\rho}[
$$

where $v_{i}^{0}=\left\{z:\left\langle v_{i}, z\right\rangle \leq 0\right\}$ denotes the polar set of $v_{i}, i=1$, 2. Since $v_{1} \neq v_{2}$ we have

$$
\mathscr{L}^{d+1}\left(v_{1}^{0} \cap v_{2}^{0} \cap \rho \mathbb{B}^{d+1}\right)<\alpha \rho^{d} \omega_{d+1} \quad \forall \rho>0
$$

for some $0<\alpha<1 / 2$. Let $\tilde{\alpha} \in] \alpha, 1 / 2[$ be fixed; if $\varepsilon$ is small enough we can find $\bar{\rho}>0$ such that

$$
\left.\mathscr{L}^{d+1}\left(\left(v_{1}^{0} \cap v_{2}^{0} \cap \rho \mathbb{B}^{d+1}\right)+2 \varepsilon \rho \mathbb{B}^{d+1}\right)<\tilde{\alpha} \rho^{d} \omega_{d+1} \quad \forall \rho \in\right] 0, \bar{\rho}[
$$

whence

$$
\limsup _{\rho \rightarrow 0^{+}} \frac{\mathscr{L}^{d+1}\left(K \cap \rho \mathbb{B}^{d+1}\right)}{\omega_{d+1} \rho^{r+1}} \leq \tilde{\alpha}<\frac{1}{2}
$$

which, recalling (6) in Theorem 2.17, proves Claim 1.

Claim 2: If $0 \in \partial^{*} K$ then $N_{K}^{F}(0) \cap \mathbb{S}^{d}=N(0)=\left\{-\nu_{K}(0)\right\}$.

Proof of Claim 2. By Claim 1 we have $N_{K}^{F}(0) \cap \mathbb{S}^{d}=N(0)=\{v\}$ for some $v \in \mathbb{S}^{d}$. For every $\varepsilon>0$ there exists $\delta_{\varepsilon}>0$ such that

$$
\left\{z \in \delta \mathbb{B}^{d+1}:\langle v, z\rangle \geq \varepsilon\|z\|\right\} \subseteq \mathbb{R}^{d+1} \backslash K \text { for any } 0<\delta<\delta_{\varepsilon} .
$$

Thus

$$
\lim _{\delta \rightarrow 0^{+}} \frac{\mathscr{L}^{d+1}\left(\delta \mathbb{B}^{d+1} \cap\left(\mathbb{R}^{d+1} \backslash K\right) \cap(-v)^{0}\right)}{\mathscr{L}^{d+1}\left(\delta \mathbb{B}^{d+1}\right)}=\frac{1}{2} .
$$

Since $0 \in \partial^{*}\left(\mathbb{R}^{d+1} \backslash K\right)$ and $\nu_{\mathbb{R}^{d+1} \backslash K}(0)=-\nu_{K}(0)$, Theorem 3.59 in [2] ensures that

$$
\lim _{\delta \rightarrow 0^{+}} \frac{\mathscr{L}^{d+1}\left(\delta \mathbb{B}^{d+1} \cap\left(\left(\mathbb{R}^{d+1} \backslash K\right) \triangle\left(\nu_{K}(0)\right)^{0}\right)\right)}{\mathscr{L}^{d+1}\left(\delta \mathbb{B}^{d+1}\right)}=0 .
$$

It is not difficult to show that equalities (23) and (24) imply that $v=-\nu_{K}(0)$, as desired. $\diamond$

In particular the implications

$$
\left\{\begin{array} { l } 
{ N _ { K } ^ { F } ( 0 ) \cap \mathbb { S } ^ { d } = \{ v \} } \\
{ \{ x _ { j } \} _ { j \in \mathbb { N } } \subseteq \partial ^ { * } K } \\
{ x _ { j } \rightarrow 0 }
\end{array} \Longrightarrow \left\{\begin{array}{l}
N(0)=\{v\} \\
N\left(x_{j}\right)=\left\{-\nu_{K}\left(x_{j}\right)\right\} \Longrightarrow \nu_{K}\left(x_{j}\right) \rightarrow-v \\
x_{j} \rightarrow 0
\end{array}\right.\right.
$$

hold because $N$ has closed graph; as a consequence we have

$$
\lim _{\rho \rightarrow 0^{+}} \sup \left\{\left|\nu_{K}(z)+v\right|: z \in \partial^{*} K \cap \rho \mathbb{B}^{d+1}\right\}=0 .
$$

Claim 3: If $N_{K}^{F}(0) \cap \mathbb{S}^{d}$ contains a unique element $v$, then $0 \in \partial^{*} K$ and $\nu_{K}(0)=-v$.

Proof of Claim 3. We have $N(0)=N_{K}^{F}(0) \cap \mathbb{S}^{d}=\{v\}$. It will be enough to show that

$$
P\left(K, \rho \mathbb{B}^{d+1}\right)=\left\|D \chi_{K}\right\|\left(\rho \mathbb{B}^{d+1}\right)>0 \quad \text { for any } \rho>0,
$$


because in this case one would get

$$
\begin{aligned}
\lim _{\rho \rightarrow 0^{+}} \frac{D \chi_{K}\left(\rho \mathbb{B}^{d+1}\right)}{\left\|D \chi_{K}\right\|\left(\rho \mathbb{B}^{d+1}\right)} & =\lim _{\rho \rightarrow 0^{+}} \frac{\int_{\partial^{*} K \cap \rho \mathbb{B}^{d+1}} \nu_{K}(z) d \mathscr{H}^{d}(z)}{\mathscr{H}^{d}\left(\partial^{*} K \cap \rho \mathbb{B}^{d+1}\right)} \\
& =-v+\lim _{\rho \rightarrow 0^{+}} f_{\partial^{*} K \cap \rho \mathbb{B}^{d+1}}\left(\nu_{K}(z)+v\right) d \mathscr{H}^{d}(z)=-v .
\end{aligned}
$$

In the previous formula the first equality comes from (4) and (5) in Theorem 2.17, while the last one is justified by (25). This would imply that $0 \in \partial^{*} K$ and $\nu_{K}(0,0)=-v$, which in turn would conclude the proof.

We have to prove (26). To this aim it will be enough to show that

$$
\mathscr{L}^{d+1}\left(\rho \mathbb{B}^{d+1} \backslash K\right)>0 \quad \text { and } \quad \mathscr{L}^{d+1}\left(K \cap \rho \mathbb{B}^{d+1}\right)>0 \quad \forall \rho>0 ;
$$

indeed, the isoperimetric inequality (cfr. Theorem 3.46 in [2]) would give

$$
\left\|D \chi_{K}\right\|\left(\rho \mathbb{B}^{d+1}\right) \geq C \cdot \min \left\{\mathscr{L}^{d+1}\left(K \cap \rho \mathbb{B}^{d+1}\right), \mathscr{L}^{d+1}\left(\rho \mathbb{B}^{d+1} \backslash K\right)\right\}^{\frac{d}{d+1}}
$$

for a suitable $C=C(d)>0$, and (26) would be proved.

Let us prove (28). Since there exists $\bar{\rho}>0$ such that

$$
\left(\rho \mathbb{B}^{d+1} \backslash K\right) \supseteq\left\{z \in \rho \mathbb{B}^{d+1}:\left\langle v_{1}, z\right\rangle \geq\|z\| / 2\right\} \text { for any } 0<\rho<\bar{\rho},
$$

we have that $\mathscr{L}^{d+1}\left(\rho \mathbb{B}^{d+1} \backslash K\right)>0$ for any $\rho>0$.

We are only left to prove the validity of the second inequality in (28). Assume by contradiction that there exists $\rho>0$ such that $\mathscr{L}^{d+1}\left(K \cap \rho \mathbb{B}^{d+1}\right)=0$; this implies that $\stackrel{\circ}{K} \cap \rho \mathbb{B}^{d+1}=\emptyset$, i.e.,

$$
K \cap \rho \mathbb{B}^{d+1}=\partial K \cap \rho \mathbb{B}^{d+1} .
$$

Since $-v \notin N_{K}^{F}(0)$ there exists a sequence $\left\{x_{n}\right\}_{n \in \mathbb{N}} \subseteq K$ such that $x_{n} \rightarrow 0$ and

$$
\left\langle-v, x_{n}\right\rangle \geq \alpha\left\|x_{n}\right\| \quad \forall n \in \mathbb{N}
$$

for some $\alpha>0$. By (29) we have $x_{n} \in \partial K$ for $n$ large enough; the $N$-regularity of $K$ ensures that for any $n$ there exists $v_{n} \in N\left(x_{n}\right)$ such that

$$
\left\langle v_{n},-x_{n}\right\rangle \leq \omega_{0}\left(\left\|x_{n}\right\|\right)
$$

for a suitable function $\omega_{0}(r)$ such that $\omega_{0}(r) / r \rightarrow 0^{+}$as $r \rightarrow 0^{+}$. On the other side, since $N$ has closed graph we have $v_{n} \rightarrow v$ and thus

$$
\left\langle-v, x_{n}\right\rangle=\left\langle v_{n}-v, x_{n}\right\rangle+\left\langle v_{n},-x_{n}\right\rangle \leq o\left(\left\|x_{n}\right\|\right)+\omega_{0}\left(\left\|x_{n}\right\|\right) .
$$

This contradicts (30) and concludes the proof.

The following result is an immediate application of Proposition 6.8.

Proposition 6.9. Let $\Omega$ be a nonempty open subset of $\mathbb{R}^{d}$ and $f: \Omega \rightarrow \mathbb{R}$ be upper semicontinuous; assume that $K:=$ hypo $f$ is $N$-regular in $\Omega \times \mathbb{R}$. Then

(1) $(x, \beta) \in \partial^{*} K \cap(\Omega \times \mathbb{R})$ if and only if $N_{K}^{F}(x, \beta) \cap \mathbb{S}^{d}$ contains a unique element; in this case we have

$$
N_{K}^{F}(x, \beta) \cap \mathbb{S}^{d}=\left\{-\nu_{K}(x, \beta)\right\} .
$$

(2) the set $\left(\partial K \backslash \partial^{*} K\right) \cap(\Omega \times \mathbb{R})$ is countably $\mathscr{H}^{d-1}$-rectifiable. 
Proof. Statement (1) follows from Proposition 6.8. As for (2), we set

$$
\begin{aligned}
& A:=\left\{(x, \beta) \in \partial K \cap(\Omega \times \mathbb{R}): \operatorname{dim} N_{K}^{F}(x, \beta) \geq 2\right\} \\
& B:=\left\{(x, \beta) \in(\partial K \backslash A) \cap(\Omega \times \mathbb{R}): N_{K}^{F}(x, \beta) \cap \mathbb{S}^{d} \text { consists of two elements }\right\} .
\end{aligned}
$$

and notice that, by statement $(1),\left(\partial K \backslash \partial^{*} K\right) \cap(\Omega \times \mathbb{R})=A \cup B$. The set $A$ is countably $\mathscr{H}^{d-1}$-rectifiable according to Theorem 1.1 and Remark 4.4, and the same holds for $B$ according to Theorem 1.1 in [31]. This concludes the proof.

\section{The UpPer estimate For the HausdorfF Dimension of $\mathscr{S}_{f}$}

The hypograph of a function $f \in \mathscr{F}(\Omega)$ is $N$-regular and, consequently, $f$ satisfies a bunch of regularity results which have been presented in the previous section. In this section, we shall give a sharp upper bound on the dimension of the singular set $\mathscr{S}_{f}$ (see Definition 5.1) for $f \in \mathscr{F}(\Omega)$. The main tool is provided by Lemma 7.2, which gives lower estimates on the total variation of $f$ around points of $\mathscr{S}_{f}$. We begin with a preliminary result.

Lemma 7.1. Let $\Omega \subseteq \mathbb{R}^{d}$ be nonempty and open and let $f \in \mathscr{F}(\Omega)$; then $f$ is locally bounded from below in $\Omega$. In particular, $f \in L_{\text {loc }}^{\infty}(\Omega)$.

Proof. The second part of the Lemma easily follows from the first one because $f$ is upper semicontinuous; therefore, it is enough to prove that $f$ is locally bounded from below in $\Omega$.

Assume by contradiction that there exists a compact set $C \subseteq \Omega$ such that $\inf _{C} f=-\infty$; then there exists $x \in C$ such that

$$
\underline{f}(x)=\liminf _{y \rightarrow x} f(x)=-\infty .
$$

Set $K:=$ hypo $f$; by Lemma 2.4 (ii) we have

$$
(x, \beta) \in \partial K \quad \forall \beta<\widetilde{f}(x)=f(x) .
$$

By Lemma 2.4 (iv) and the assumption that $f \in \mathscr{F}(\Omega)$ there exist $C, \theta$ such that the following holds: for any $\beta<f(x)$ there exists $v_{\beta} \in \mathbb{S}^{d-1}$ such that

$$
\left(v_{\beta}, 0\right) \in \hat{\mathscr{N}}_{K}^{C, \theta}(x, \beta) .
$$

This means that for any $\beta<f(x)$ it holds

$$
\left\langle v_{\beta}, y-x\right\rangle \leq C\left(\|y-x\|^{1+\theta}+\left|\beta_{y}-\beta\right|^{1+\theta}\right) \quad \forall y \in \Omega, \beta_{y} \leq f(y) .
$$

Let us fix a decreasing sequence $\left\{\beta_{n}\right\}_{n}$ such that $\beta_{n}<f(x)$ for any $n, \beta_{n} \rightarrow-\infty$ and $v_{n}:=$ $v_{\beta_{n}} \rightarrow v \in \mathbb{S}^{d-1}$. Choose also $\delta>0$ so small that

$$
C \delta^{1+\theta} \leq \delta / 4 \text { and } y:=x+\delta v \in \Omega
$$

and $\bar{n} \in \mathbb{N}$ so large that

$$
\left\langle v_{\bar{n}}, v\right\rangle \geq 1 / 2 \text { and } \beta_{\bar{n}} \leq f(y) .
$$

We can then use (31) with $\beta=\beta_{y}=\beta_{\bar{n}}$ to get

$$
\delta / 2 \leq\left\langle v_{\bar{n}}, \delta v\right\rangle \leq C \delta^{1+\theta} \leq \delta / 4
$$

which gives a contradiction and proves the Lemma. 
Lemma 7.2. Let $\Omega \subseteq \mathbb{R}^{d}$ be nonempty and open and let $f \in \mathscr{F}(\Omega)$. Let $x \in \mathscr{S}_{f}$ be such that $N_{\text {hypo } f}^{F}(x, \underline{f}(x))=\mathbb{R}^{+}(v, 0)$ for some $v \in \mathbb{S}^{d-1}$. Then there exists $\delta_{0}=\delta_{0}(x)>0$ such that

$$
\|D f\|(\operatorname{Sq}(x, \delta)) \geq 2^{d-2} \cdot \delta^{d-\frac{\theta}{1+\theta}} \quad \text { for all } 0<\delta<\delta_{0} .
$$

Proof. Lemma 7.1 ensures that $\underline{f}(x)>-\infty$; in particular, without loss of generality we may assume that

$$
x=0 \in \Omega, \quad \underline{f}(x)=0 \quad \text { and } \quad N_{\text {hypo } f}^{F}(0,0)=\mathbb{R}^{+}\left(e_{1}, 0\right) .
$$

For any $\delta>0$ define

$$
\begin{aligned}
& R_{\delta}:=\left\{y=\left(y_{1}, \ldots, y_{d}\right) \in \operatorname{Sq}(0, \delta): \frac{3}{4} \delta<y_{1}<\delta\right\}, \\
& S_{\delta}:=\left\{y=\left(y_{1}, \ldots, y_{d}\right) \in \operatorname{Sq}(0, \delta):-\delta<y_{1}<-\delta / 2\right\} .
\end{aligned}
$$

Claim: There exist $\delta_{1}, \delta_{2}>0$ such that

$$
f(y) \leq-\frac{1}{2} \delta^{\frac{1}{1+\theta}}, \quad \forall y \in R_{\delta}, \delta<\delta_{1}
$$

and

$$
f(y)>0 \quad \forall y \in S_{\delta}, \delta<\delta_{2} .
$$

Proof of Claim: Let us prove (33). For $y \in R_{\delta}$ we have

$$
\frac{3}{4} \delta<\left\langle\left(e_{1}, 0\right),(y, \beta)\right\rangle \leq C \cdot\left(\|y\|^{1+\theta}+|\beta|^{1+\theta}\right) \quad \forall \beta \leq f(y)
$$

whence

$$
\frac{3}{4} \delta \leq C\left(d^{\frac{1+\theta}{2}} \delta^{1+\theta}+|\beta|^{1+\theta}\right), \quad \forall \beta \leq f(y) .
$$

Notice that, for $\delta$ small enough, it holds

$$
f(y)<0 \quad \text { for any } y \in R_{\delta}
$$

indeed, by contradiction, if $f(y) \geq 0$ one could choose $\beta=0$, thus violating (35) for $\delta$ sufficiently small. Formula (33) easily follows for a small enough $\delta_{1}$ on taking $\beta=f(y)<0$ in (35).

Let us prove (34). Assume by contradiction that there exist sequences $\left\{\delta_{n}\right\}_{n}$ and $\left\{y^{n}\right\}_{n}$ such that

$$
\delta_{n} \rightarrow 0^{+}, \quad y^{n} \in S_{\delta_{n}} \quad \text { and } \quad f\left(y^{n}\right) \leq 0 .
$$

Since $y^{n} \rightarrow 0$ and $\underline{f}(0)=0$ we get $\lim _{n \rightarrow \infty} f\left(y^{n}\right)=\underline{f}(0)=0$. Hence, by the upper semicontinuity of $N$, there exists a sequence $\left\{\left(v^{n}, \alpha^{n}\right)\right\}_{n \in \mathbb{N}}$ such that $\left(v^{n}, \alpha^{n}\right) \in N\left(y^{n}, f\left(y^{n}\right)\right)$ and $\left(v^{n}, \alpha^{n}\right) \rightarrow\left(e_{1}, 0\right)$ (we recall that if $\left(v^{n}, \alpha^{n}\right) \in N\left(y^{n}, f\left(y^{n}\right)\right)$ then $\left.\left\|\left(v^{n}, \alpha^{n}\right)\right\|=1\right)$; in particular, $v^{n} \rightarrow e_{1}$. Moreover, for all $\beta \leq 0=f(0)$ it holds

$$
\left\langle\left(v^{n}, \alpha^{n}\right),(0, \beta)-\left(y^{n}, f\left(y^{n}\right)\right)\right\rangle \leq C \cdot\left(\left\|y^{n}\right\|^{1+\theta}+\left|\beta-f\left(y^{n}\right)\right|^{1+\theta}\right) .
$$

Since $f\left(y^{n}\right) \leq \underline{f}(0)=0$ we can choose $\beta=f\left(y^{n}\right)$ in the above inequality and get

$$
\left\langle v^{n},-y^{n}\right\rangle \leq C \cdot\left\|y^{n}\right\|^{1+\theta} .
$$

Thus

$$
\frac{\delta_{n}}{2}-\left\|v^{n}-e_{1}\right\| \sqrt{d} \delta_{n} \leq\left\langle e_{1},-y^{n}\right\rangle+\left\langle v^{n}-e_{1},-y^{n}\right\rangle=\left\langle v^{n},-y^{n}\right\rangle \leq C d^{\frac{1+\theta}{2}} \delta_{n}^{1+\theta} .
$$


Dividing both sides by $\delta_{n}$ and passing to the limit as $n \rightarrow \infty$ we obtain a contradiction. This concludes the proof of the Claim.

The Claim allows us to conclude: indeed, for any $\delta<\delta_{0}:=\min \left\{\delta_{1}, \delta_{2}\right\}$ and any $z \in(-\delta, \delta)^{d-1}$ we get

$$
\left.\left|f\left(y_{a}, z\right)-f\left(y_{b}, z\right)\right| \geq \frac{1}{2} \delta^{\frac{1}{1+\theta}} \quad \forall y_{a} \in\right] \frac{3}{4} \delta, \delta\left[, y_{b} \in\right]-\delta,-\delta / 2[.
$$

By virtue of Lemma 2.18, for any $z \in(-\delta, \delta)^{d-1}$ there exist $\left.y_{a}(z) \in\right] \frac{3}{4} \delta, \delta\left[\right.$ and $\left.y_{b}(z) \in\right]-\delta,-\delta / 2[$ such that

$$
\left\|D f_{z}\right\|(-\delta, \delta) \geq\left|f\left(y_{a}(z), z\right)-f\left(y_{b}(z), z\right)\right| \geq \frac{1}{2} \delta^{\frac{1}{1+\theta}}
$$

where $f_{z}:=f(\cdot, z)$. By $[2$, Theorem 3.103] we obtain

$$
\begin{aligned}
\|D f\|(\operatorname{Sq}(0, \delta)) & \geq \int_{]-\delta, \delta[d-1}\left\|D_{e_{1}} f\right\|(z+]-\delta, \delta\left[e_{1}\right) d z \\
& =\int_{]-\delta, \delta[d-1}\left\|D f_{z}\right\|(-\delta, \delta) d z \\
& \geq(2 \delta)^{d-1} \cdot \frac{1}{2} \delta^{\frac{1}{1+\theta}}=2^{d-2} \delta^{d-\frac{\theta}{1+\theta}},
\end{aligned}
$$

where we have denoted by $D_{e_{1}} f$ the distributional derivative of $f$ along $e_{1}$ and by $\left.z+\right]-\delta, \delta\left[e_{1}\right.$ the line segment joining $(-\delta, z)$ and $(\delta, z)$. This concludes the proof of the Lemma.

We are ready to prove the third main result of the paper.

Proof of Theorem 1.3. Set $K=$ hypo $f$; by Proposition 6.1, without loss of generality we may assume $U=\Omega$ and $f \in B V(\Omega)$.

Let $\pi: \mathbb{R}^{d+1} \rightarrow \mathbb{R}^{d}$ be the projection $\pi\left(x_{1}, \ldots, x_{d+1}\right)=\left(x_{1}, \ldots, x_{d}\right)$. The set $\left(\partial K \backslash \partial^{*} K\right) \cap$ $(\Omega \times \mathbb{R})$ is countably $\mathscr{H}^{d-1}$-rectifiable by Proposition $6.9(2)$; since $\pi$ is Lipschitz continuous, also $\mathscr{S}_{f} \backslash \pi\left(\partial^{*} K\right)$ is countably $\mathscr{H}^{d-1}$-rectifiable because

$$
\mathscr{S}_{f} \backslash \pi\left(\partial^{*} K\right) \subseteq \Omega \backslash \pi\left(\partial^{*} K\right)=\pi\left(\left(\partial K \backslash \partial^{*} K\right) \cap(\Omega \times \mathbb{R})\right) .
$$

In particular $\mathscr{H}^{d-\frac{\theta}{1+\theta}}\left(\Omega \backslash \pi\left(\partial^{*} K\right)\right)=0$ and it will be enough to show that

$$
\mathscr{H}^{d-\frac{\theta}{1+\theta}}\left(\mathscr{S}_{f} \cap \pi\left(\partial^{*} K\right)\right)<+\infty .
$$

According to Proposition 6.9 (1) and the estimates given by Lemma 7.2, there exists a constant $C>0$ depending only on $d, \theta$ such that

$$
\limsup _{\delta \rightarrow 0^{+}} \frac{\|D f\|\left(x+\delta \mathbb{B}^{d}\right)}{\omega_{d-\frac{\theta}{1+\theta}} \delta^{d-\frac{\theta}{1+\theta}}} \geq C
$$

for all $x \in \mathscr{S}_{f} \cap \pi\left(\partial^{*} K\right)$. By Theorem 2.10 we get

$$
\|D f\|\left(\mathscr{S}_{f} \cap \pi\left(\partial^{*} K\right)\right) \geq C \mathscr{H}^{d-\frac{\theta}{1+\theta}}\left(\mathscr{S}_{f} \cap \pi\left(\partial^{*} K\right)\right)
$$

and we can conclude because $\|D f\|(\Omega)<+\infty$. 
The following result shows that the bound $\operatorname{dim}_{\mathscr{H}} \mathscr{S}_{f} \leq d-\frac{\theta}{1+\theta}$ is sharp. We will focus on the case $d=1, \theta=1$, i.e., when hypo $f$ satisfies an uniform external ball condition, but our construction can be easily adapted to cover more general cases.

Proposition 7.3. For every $\varepsilon>0$ there exists a continuous map $f:[0,1] \rightarrow \mathbb{R}$ such that for any $x \in[0,1]$ there exist $v_{x} \in N_{\text {hypo } f}^{F}(x, f(x)) \cap \mathbb{S}^{1}$ with

$$
\left\langle v_{x}, y-x\right\rangle \leq|y-x|^{2}+|f(y)-f(x)|^{2} \quad \text { for every } y \in[0,1]
$$

and $\operatorname{dim}_{\mathscr{H}} \mathscr{S}_{f} \geq \frac{1}{2}-\varepsilon$.

Proof. Fix $\varepsilon>0$ and let $\lambda \in] 0,1 / 4[$ be such that

$$
\frac{1}{2}-\varepsilon \leq \log _{\lambda} \frac{1}{2}=\log _{1 / \lambda} 2<\frac{1}{2} .
$$

Consider the Cantor set $C_{\lambda}$ constructed in this way:

Step 0: remove from $I:=[0,1]$ an open interval $I_{1}^{0}$ of lenght $1-2 \lambda$ centered at the middle point of $I$, i.e. $1 / 2$. We are left with 2 closed intervals of lenght $\lambda$.

Step 1: From each of the two remaining intervals, remove an open interval of lenght $\lambda(1-2 \lambda)$ centered on its midpoint. In this way we are removing two intervals $I_{1}^{1}$ and $I_{2}^{1}$ and we are left with $2^{2}=4$ closed intervals of lenght $\lambda^{2}$.

Step $n$ : From each of the $2^{n}$ remaining closed intervals of lenght $\lambda^{n}$, remove open intervals $I_{1}^{n}, \ldots, I_{2^{n}}^{n}$ of lenght $\lambda^{n}(1-2 \lambda)$ centered in their midpoints. We are left with $2^{n+1}$ closed intervals of lenght $\lambda^{n+1}$.

We define $C_{\lambda}$ as the intersection of all the closed intervals we are left with at each step or, equivalently,

$$
C_{\lambda}=[0,1] \backslash \bigcup_{n=0}^{\infty} \bigcup_{i=1}^{2^{n}} I_{i}^{n}
$$

It is well known (see e.g. [23]) that

$$
\operatorname{dim}_{\mathscr{H}} C_{\lambda}=\log _{1 / \lambda} 2 \geq \frac{1}{2}-\varepsilon .
$$

We are going to provide a continuous function $f:[0,1] \rightarrow \mathbb{R}$ such that $\mathscr{S}_{f}=C_{\lambda}$ and for every $x \in[0,1]$ there exists $v_{x} \in N_{\text {hypo } f}^{F}(x, f(x)) \cap \mathbb{S}^{1}$ with

$$
\left\langle v_{x}, y-x\right\rangle \leq|y-x|^{2}+|f(y)-f(x)|^{2} \quad \text { for every } y \in[0,1] \text {. }
$$

Let $\left.\left\{b_{i}^{n}\right\}_{i=1, \ldots, 2^{n}}^{n \in \mathbb{N}} \subseteq\right] 0,1[$ be such that

$$
\left.I_{i}^{n}=\right] b_{i}^{n}-\lambda^{n}(1-2 \lambda), b_{i}^{n}[
$$

and define

$$
g(x):= \begin{cases}\left(-\sqrt{1-\left(x-b_{i}^{n}+1\right)^{2}}\right)^{\prime}=\frac{x-b_{i}^{n}+1}{\sqrt{1-\left(x-b_{i}^{n}+1\right)^{2}}} & \text { if } x \in I_{i}^{n}, \\ 0 & \text { otherwise. }\end{cases}
$$


We have that $g(x) \geq 0$ and

$$
\begin{aligned}
\int_{I_{i}^{n}} g(x) d x & =\left[-\sqrt{1-\left(x-b_{i}^{n}+1\right)^{2}}\right]_{x=b_{i}^{n}-\lambda^{n}(1-2 \lambda)}^{x=b_{i}^{n}} \\
& =\sqrt{2 \lambda^{n}(1-2 \lambda)-\lambda^{2 n}(1-2 \lambda)^{2}} \leq \sqrt{2(1-2 \lambda)} \lambda^{n / 2} \leq \sqrt{2} \lambda^{n / 2} .
\end{aligned}
$$

This implies that $g \in L^{1}(0,1)$ because

$$
\int_{0}^{1} g(x) d x=\sum_{n=0}^{\infty} \sum_{i=1}^{2^{n}} \int_{I_{i}^{n}} g(x) d x \leq \sqrt{2} \sum_{n=0}^{\infty} \sum_{i=1}^{2^{n}} \lambda^{n / 2}=\sqrt{2} \sum_{n=0}^{\infty}(2 \sqrt{\lambda})^{n}
$$

which is finite because $\lambda<1 / 4$. Thus the function $f:[0,1] \rightarrow \mathbb{R}$ defined by

$$
f(x):=\int_{0}^{x} g(t) d t
$$

belongs to $A C([0,1])$ and, in particular, is $B V$ and continuous on $[0,1]$, and of class $C^{1}$ on each interval $I_{i}^{n}$. Let $y \in I_{i}^{n}$; we have

$$
f(y)-f\left(b_{i}^{n}\right)=\int_{b_{i}^{n}}^{y} g(x) d x=-\sqrt{1-\left(y-b_{i}^{n}+1\right)^{2}}
$$

whence

$$
\left(f(y)-f\left(b_{i}^{n}\right)\right)^{2}+\left(y-b_{i}^{n}+1\right)^{2}=1 \quad \forall y \in I_{i}^{n} .
$$

Thus the graph of $f$ restricted to each $I_{i}^{n}$ corresponds to an arc of unit circle centered at $\left(b_{i}^{n}-1, f\left(b_{i}^{n}\right)\right)$ and it is not difficult to see that hypo $f$ satisfies an external ball condition of radius 1 . Moreover

$$
\{(-1,0)\} \in N_{\text {hypo } f}^{F}\left(b_{i}^{n}, f\left(b_{i}^{n}\right)\right) \cap \mathbb{S}^{1},
$$

because $g(y) \rightarrow+\infty$ as $y \rightarrow\left(b_{i}^{n}\right)^{-}$. So $\mathscr{S}_{f} \supseteq\left\{b_{i}^{n}: n \in \mathbb{N}, i=1, \ldots, 2^{n}\right\}$ and, since $\mathscr{S}_{f}$ is closed and

$$
\overline{\left\{b_{i}^{n}: n \in \mathbb{N}, i=1, \ldots, 2^{n}\right\}}=C_{\lambda},
$$

we have $\mathscr{S}_{f} \supseteq C_{\lambda}$. Moreover we have also $\mathscr{S}_{f} \subseteq C_{\lambda}$ because $\mathscr{S}_{f} \cap I_{i}^{n}=\emptyset$ for every $n \in \mathbb{N}$, $i=1, \ldots, 2^{n} ;$ thus $\mathscr{S}_{f}=C_{\lambda}$, as desired.

Remark 7.4. The previous result corrects Example 5.2 in [15].

\section{Appendix: an eXample in Optimal Control}

We resume the discussion of the example described in the Introduction. We were considering the constant control system (3) together with the target $\mathcal{T}=$ epi $f$, where

$$
f(x)=\chi_{]-\infty, 0]}(x)-|x|^{2 / 3} \chi_{] 0,+\infty[}(x) .
$$

The minimum time $T$ to reach target $\mathcal{T}$ subject to the above control system can be explicitly computed. Given $(x, y) \notin \overline{\mathcal{T}}$, we have

$$
T(x, y)= \begin{cases}1-y & \text { if } x \leq 0 \\ -x^{2 / 3}-y, & \text { if } x>0\end{cases}
$$


Clearly, $T$ is discontinuous on the set $\left\{(0, y) \in \mathbb{R}^{2}: y \leq 0\right\}$. Moreover, for every $r>0$, the closure of the sublevel $\{(x, y): T(x, y)<r\}$ does not satisfy an exterior sphere condition at $(0,-r)$. Hence, such condition does not hold for the hypograph of $T$ at the point $(0,-r, r)$ either. Similarly, the exterior sphere condition does not hold for epi $f$ at the origin.

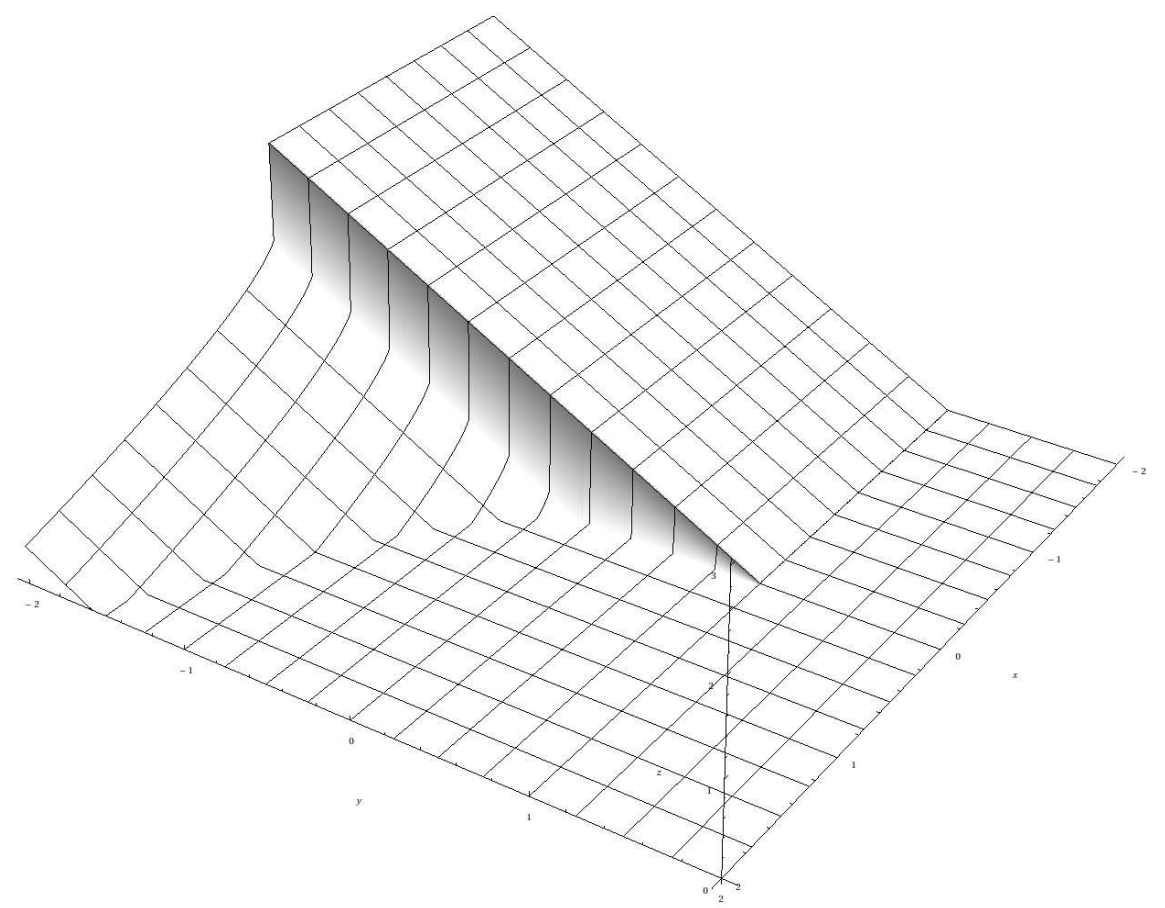

FiguRE 3. The graph of the minimum time function $T$.

However, $T$ belongs to the class $\mathscr{F}(\Omega)$ for $\Omega:=\mathbb{R}^{2} \backslash \overline{\mathcal{T}}$. Indeed, one can first see from (36) that $T$ is upper-semicontinuous on $\Omega$, thus we need only to check that hypo $T \in \mathscr{F} \Omega \times \mathbb{R}$. To this aim, it suffices to prove that $\overline{\text { hypo } T_{\mid \Omega}} \in \mathscr{F}$.

Define

$$
\begin{aligned}
& S_{1}:=\left\{(x, y, z) \in \mathbb{R}^{3}: z+y-1 \leq 0 \text { and } x \leq 0\right\} \\
& S_{2}:=\left\{(x, y, z) \in \mathbb{R}^{3}:(z+y)^{3}+x^{2} \leq 0 \text { and } x \geq 0\right\} \\
& S_{3}:=\left\{(x, y, z) \in \mathbb{R}^{3}: x \leq 0 \text { or } y \leq-x^{2 / 3}\right\}=\left\{(x, y, z) \in \mathbb{R}^{3}: x \leq \max \{0, y\}^{3 / 2}\right\} \\
& S_{4}:=\left\{(x, y, z) \in \mathbb{R}^{3}: y \leq 1\right\} .
\end{aligned}
$$

and notice that

$$
\overline{\text { hypo } T_{\mid \Omega}}=\left(S_{1} \cup S_{2}\right) \cap S_{3} \cap S_{4} .
$$

Since the class $\mathscr{F}$ is closed under intersection, in order to prove that hypo $\overline{T_{\mid \Omega}} \in \mathscr{F}$ it is enough to prove that $S_{1} \cup S_{2}, S_{3}$ and $S_{4}$ belong to $\mathscr{F}$. 
Using the fact that the map $y \mapsto \max \{0, y\}^{3 / 2}$ is of class $C^{1,1 / 2}$, it is not difficult to show that

$$
\mathscr{N}_{S_{3}}^{C, 1 / 2}(x, y, z) \neq\{0\} \quad \forall(x, y, z) \in \partial S_{3}
$$

for a suitable $C=C\left(S_{3}\right)>0$; in particular, $S_{3} \in \mathscr{F}$. The halfspace $S_{4}$ clearly belongs to $\mathscr{F}$. Finally, under the linear invertible change of coordinates

$$
(u, v, w)=F(x, y, z)=(x, z+y, z-y),
$$

under which $\mathscr{F}$ is clearly closed, we have

$$
\begin{aligned}
& F\left(S_{1}\right)=\left\{(u, v, w) \in \mathbb{R}^{3}: v \leq 1 \text { and } u \leq 0\right\} \\
& F\left(S_{2}\right)=\left\{(u, v, w) \in \mathbb{R}^{3}: v^{3} \leq-u^{2} \text { and } u \leq 0\right\} .
\end{aligned}
$$

This means that $F\left(S_{1} \cup S_{2}\right)=($ hypo $g) \times \mathbb{R}$, where

$$
g(v)= \begin{cases}-\infty, & v>1 \\ 0, & v \in[0,1] \\ |v|^{3 / 2}, & v<0\end{cases}
$$

In order to prove that $F\left(S_{1} \cup S_{2}\right) \in \mathscr{F}$ it is enough to show that hypo $g \in \mathscr{F} \mathbb{R}^{2}$, and this can be easily checked using the fact that $\left.\left.g \in C^{1,1 / 2}(]-\infty, 1\right]\right)$. We have that hypo $g$ is $N$-regular and

$$
\mathscr{N}_{\text {hypo } g}^{C, 1 / 2}(u, v) \neq\{0\} \quad \forall(u, v) \in \text { ohypo } g .
$$

This is enough to conclude.

\section{REFERENCES}

[1] Jean-Pierre Aubin and Arrigo Cellina, Differential inclusions, Grundlehren der Mathematischen Wissenschaften [Fundamental Principles of Mathematical Sciences], vol. 264, Springer-Verlag, Berlin, 1984. Set-valued maps and viability theory. MR755330 (85j:49010)

[2] Luigi Ambrosio, Nicola Fusco, and Diego Pallara, Functions of bounded variation and free discontinuity problems, Oxford Mathematical Monographs, The Clarendon Press Oxford University Press, New York, 2000. MR1857292 (2003a:49002)

[3] Martino Bardi and Italo Capuzzo-Dolcetta, Optimal control and viscosity solutions of Hamilton-JacobiBellman equations, Systems \& Control: Foundations \& Applications, Birkhäuser Boston Inc., Boston, MA, 1997. With appendices by Maurizio Falcone and Pierpaolo Soravia. MR1484411 (99e:49001)

[4] Guy Barles, Pierre Cardaliaguet, Olivier Ley, and Aurélien Monteillet, Uniqueness results for nonlocal Hamilton-Jacobi equations, Journal of Functional Analysis 257 (2009), no. 5, 1261-1287, DOI 10.1016/j.jfa.2009.04.014.

[5] Joël Benoist, The size of the Dini subdifferential, Proc. Amer. Math. Soc. 129 (2001), no. 2, 525-530, DOI 10.1090/S0002-9939-00-05549-0. MR1707505 (2001e:26006)

[6] F. Bernard and L. Thibault, Uniform prox-regularity of functions and epigraphs in Hilbert spaces, Nonlinear Anal. 60 (2005), no. 2, 187-207, DOI 10.1016/j.na.2004.04.015. MR2101873 (2005h:49035)

[7] James V. Burke, Adrian S. Lewis, and Michael L. Overton, Approximating subdifferentials by random sampling of gradients, Math. Oper. Res. 27 (2002), no. 3, 567-584, DOI 10.1287/moor.27.3.567.317. MR1926659 (2003g:49024)

[8] Annamaria Canino, On p-convex sets and geodesics, J. Differential Equations 75 (1988), no. 1, 118-157, DOI 10.1016/0022-0396(88)90132-5. MR957011 (90b:58044) 
[9] Piermarco Cannarsa and Carlo Sinestrari, Convexity properties of the minimum time function, Calc. Var. Partial Differential Equations 3 (1995), no. 3, 273-298, DOI 10.1007/s005260050016. MR1385289 (97f:49032)

[10] _ Semiconcave functions, Hamilton-Jacobi equations, and optimal control, Progress in Nonlinear Differential Equations and their Applications, 58, Birkhäuser Boston Inc., Boston, MA, 2004. MR2041617 (2005e:49001)

[11] Piermarco Cannarsa and Khai T. Nguyen, Exterior sphere condition and time optimal control for differential inclusions, SIAM J. Control Optim., to appear.

[12] Miroslav Chlebík, Andrea Cianchi, and Nicola Fusco, The perimeter inequality under Steiner symmetrization: cases of equality, Ann. of Math. (2) 162 (2005), no. 1, 525-555, DOI 10.4007/annals.2005.162.525. MR2178968 (2006m:49032)

[13] Francis H. Clarke, Optimization and nonsmooth analysis, 2nd ed., Classics in Applied Mathematics, vol. 5, Society for Industrial and Applied Mathematics (SIAM), Philadelphia, PA, 1990. MR1058436 (91e:49001)

[14] Francis H. Clarke, Yuri S. Ledyaev, Ronald J. Stern, and Peter R. Wolenski, Nonsmooth analysis and control theory, Graduate Texts in Mathematics, vol. 178, Springer-Verlag, New York, 1998. MR1488695 (99a:49001)

[15] Giovanni Colombo and Antonio Marigonda, Differentiability properties for a class of non-convex functions, Calc. Var. Partial Differential Equations 25 (2006), no. 1, 1-31, DOI 10.1007/s00526-005-0352-7. MR2183853 (2006h:49027)

[16] _ Singularities for a class of non-convex sets and functions, and viscosity solutions of some HamiltonJacobi equations, J. Convex Anal. 15 (2008), no. 1, 105-129. MR2389006 (2009i:49023)

[17] Giovanni Colombo, Antonio Marigonda, and Peter R. Wolenski, Some new regularity properties for the minimal time function, SIAM J. Control Optim. 44 (2006), no. 6, 2285-2299 (electronic), DOI 10.1137/050630076. MR2248184 (2008d:49021)

[18] _ The Clarke generalized gradient for functions whose epigraph has positive reach (2011), submitted.

[19] Giovanni Colombo and Khai T. Nguyen, On the structure of the minimum time function, SIAM J. Control Optim. 48 (2010), no. 7, 4776-4814, DOI 10.1137/090774549. MR2720234

[20] _ On the Minimum Time Function Around the Origin, preprint 2011, arXiv:1110.0088.

[21] Giovanni Colombo and Lionel Thibault, Prox-regular sets and applications, in Handbook of Nonconvex Analysis, D.Y. Gao, D. Motreanu eds., International Press, Somerville, MA, 2010.

[22] Lawrence C. Evans and Ronald F. Gariepy, Measure theory and fine properties of functions, Studies in Advanced Mathematics, CRC Press, Boca Raton, FL, 1992. MR1158660 (93f:28001)

[23] Kenneth J. Falconer, The geometry of fractal sets., Cambridge Tracts in Mathematics, vol. 85, Cambridge University Press, Cambridge, 1986. MR0867284 (88d:28001)

[24] Herbert Federer, Curvature measures, Trans. Amer. Math. Soc. 93 (1959), 418-491. MR0110078 (22 \#961)

[25] _ Geometric measure theory, Die Grundlehren der mathematischen Wissenschaften, Band 153, Springer-Verlag New York Inc., New York, 1969. MR0257325 (41 \#1976)

[26] Nicola Fusco, Maria Stella Gelli, and Giovanni Pisante, On a Bonnesen type inequality involving the spherical deviation, preprint 2011.

[27] Enrico Giusti, Minimal surfaces and functions of bounded variation, Monographs in Mathematics, vol. 80, Birkhäuser Verlag, Basel, 1984. MR775682 (87a:58041)

[28] Mario Miranda, Superfici cartesiane generalizzate ed insiemi di perimetro localmente finito sui prodotti cartesiani., Ann. Scuola Norm. Sup. Pisa 18 (1964), 515-542 (Italian). MR0174706 (30 \#4906)

[29] Boris S. Mordukhovich, Variational analysis and generalized differentiation. I, Grundlehren der Mathematischen Wissenschaften [Fundamental Principles of Mathematical Sciences], vol. 330, Springer-Verlag, Berlin, 2006. Basic theory. MR2191744 (2007b:49003a)

[30] Khai T. Nguyen, Hypographs satisfying an external sphere condition and the regularity of the minimum time function, J. Math. Anal. Appl. 372 (2010), 611-628, DOI 10.1016/j.jmaa.2010.07.010. 
[31] Khai T. Nguyen and Davide Vittone, Rectifiability of siguralities of non-lipschitz functions, J. of Convex Analysis 19 (2012), no. 1, to appear.

[32] Chadi Nour, Ronald J. Stern, and Jean Takche, Proximal smoothness and the exterior sphere condition, J. Convex Anal. 16 (2009), no. 2, 501-514. MR2559957 (2010j:49028)

[33] _ The union of uniform closed balls conjecture, Control Cybernet. 38 (2009), no. 4B, 1525-1534. MR2760918

[34] - The $\theta$-exterior sphere condition, $\phi$-convexity, and local semiconcavity, Nonlinear Anal. 73 (2010), no. 2, 573-589, DOI 10.1016/j.na.2010.04.001. MR2650840

[35] _ Validity of the union of uniform closed balls conjecture, J. Convex Anal. 18 (2011), no. 2, 589-600.

[36] - Generalized exterior sphere conditions and $\phi$-convexity, Discrete Contin. Dyn. Syst. 29 (2011), no. 2, 615-622, DOI 10.3934/dcds.2011.29.615. MR2728474

[37] René A. Poliquin and R. Tyrrell Rockafellar, Prox-regular functions in variational analysis, Trans. Amer. Math. Soc. 348 (1996), no. 5, 1805-1838, DOI 10.1090/S0002-9947-96-01544-9. MR1333397 (96h:49039)

[38] Ludovic Rifford, Refinement of the Benoist theorem on the size of Dini subdifferentials, Commun. Pure Appl. Anal. 7 (2008), no. 1, 119-124, DOI 10.3934/cpaa.2008.7.119. MR2358358 (2008k:49036)

[39] R. Tyrrell Rockafellar, Convex analysis, Princeton Mathematical Series, No. 28, Princeton University Press, Princeton, N.J., 1970. MR0274683 (43 \#445)

[40] _ Clarke's tangent cones and the boundaries of closed sets in $\mathbf{R}^{n}$, Nonlinear Anal. 3 (1979), no. 1, 145-154 (1978), DOI 10.1016/0362-546X(79)90044-0. MR520481 (80d:49032)

[41] R. Tyrrell Rockafellar and Roger J.-B. Wets, Variational analysis, Grundlehren der Mathematischen Wissenschaften [Fundamental Principles of Mathematical Sciences], vol. 317, Springer-Verlag, Berlin, 1998. MR1491362 (98m:49001)

[42] Cédric Villani, Optimal transport, old and new, Grundlehren der mathematischen Wissenschaften [Fundamental Principles of Mathematical Sciences], vol. 338, Springer-Verlag, Berlin, 2009. 2 M1491362 (98m:49001)

Antonio Marigonda: Department of Computer Sciences, University of Verona. Strada Le Grazie 15, I-37134 VERONA, ItALy.

E-mail address: antonio.marigonda@univr.it

Khai T. Nguyen: Dipartimento di Matematica, Università di Padova. Via Trieste 63, I-35121 PADOVA, ITALY.

E-mail address: khai@math.unipd.it

Davide Vittone: Dipartimento di Matematica, Università di Padova. Via Trieste 63, I-35121 PADOVA, ITALY.

E-mail address: vittone@math.unipd.it 